Tunable Thermal Conductivity via Domain Structure Engineering in Ferroelectric Thin Films: A Phase-Field Simulation

\author{
Jian-Jun Wang $^{1 *}$, Yi Wang ${ }^{1}$, Jon F. Ihlefeld ${ }^{2}$, Patrick E. Hopkins ${ }^{3}$, Long-Qing Chen ${ }^{1}$ \\ ${ }^{1}$ Department of Materials Science and Engineering, The Pennsylvania State University, \\ University Park, Pennsylvania 16802, USA \\ ${ }^{2}$ Sandia National Laboratories, Albuquerque, New Mexico 87185, USA \\ ${ }^{3}$ Department of Mechanical and Aerospace Engineering, University of Virginia, Charlottesville, \\ Virginia 22904, USA
}

\begin{abstract}
Effective thermal conductivity as a function of domain structure is studied by solving the heat conduction equation using a spectral iterative perturbation algorithm in materials with inhomogeneous thermal conductivity distribution. Using this proposed algorithm, the experimentally measured effective thermal conductivities of domain-engineered $\{001\}_{p}-\mathrm{BiFeO}_{3}$ thin films are quantitatively reproduced. In conjunction with two other testing examples, this proposed algorithm is proven to be an efficient tool for interpreting the relationship between the effective thermal conductivity and micro-/domain-structures. By combining this algorithm with the phase-field model of ferroelectric thin films, the effective thermal conductivity for $\mathrm{PbZr}_{1 \text { - }}$ ${ }_{x} \mathrm{Ti}_{x} \mathrm{O}_{3}$ films under different composition, thickness, strain, and working conditions is predicted. It is shown that the chemical composition, misfit strain, film thickness, film orientation, and a Piezoresponse Force Microscopy tip can be used to engineer the domain structures and tune the effective thermal conductivity. Therefore, we expect our findings will stimulate future theoretical, experimental and engineering efforts on developing devices based on the tunable effective thermal conductivity in ferroelectric nanostructures.
\end{abstract}




\section{Acta Materialia}

\section{Introduction}

The ability to deterministically control the thermal conductivity for semiconductors is of fundamental importance in fields of phononics and thermoelectrics [1,2]. Enhancing scattering during phonon transport provides a means to achieve this control. This can be achieved by engineering the high-densities of interfaces in nanostructured materials such as superlattices [36], nanowires [7,8], quantum dots [9-12], and nanocomposites [13-17]. When the size of the nanostructure is smaller than the phonon mean free path, phonons can be strongly scattered, giving rise to a decrease in the thermal conductivity.

In ferroelectrics, such as $\mathrm{BaTiO}_{3}$ [18] and $\mathrm{KDP}$ [19] single crystals and $\mathrm{BiFeO}_{3}$ [20] and $\mathrm{PbZr}_{1-x} \mathrm{Ti}_{x} \mathrm{O}_{3}(\mathrm{PZT})$ [21] thin films, it was found that the ferroelastic domain walls acted as interfaces that can scatter phonons resulting in a net decrease in the effective thermal conductivity. For thin films, the size of ferroelectric domains ranges from several nanometers to hundreds of nanometers depending on the chemical composition, material size, and mechanical and electric boundary conditions of the film [22], which is generally compatible with those phonon mean free paths that carry thermal energy at room temperature [23-26]. Recently, altering the effective thermal conductivity via domain structures engineering either by controlling the film-growing conditions [20] or by applying an electric field [21] has been demonstrated.

In this work, taking $\mathrm{BiFeO}_{3}$ and $\mathrm{PbZr}_{1-x} \mathrm{Ti}_{x} \mathrm{O}_{3}$ thin films as examples, we demonstrate that the effective thermal conductivity can be tuned by engineering the ferroelectric domain structure. In order to achieve this, we employ the phase-field model of ferroelectric thin films (see Sec. 2A) to evolve the domain structures of $\mathrm{PbZr}_{1-x} \mathrm{Ti}_{x} \mathrm{O}_{3}$ [27-29]. We assume that the domain and domain wall can be regarded as two identities with different thermal conductivities 


\section{Acta Materialia}

[20,21]. In order to obtain the effective thermal conductivity as function of domain structure, we solve the stationary heat conduction equation with an inhomogeneous thermal conductivity distribution using a spectral iterative perturbation (SIP) method [30-33], as shown in Sec. $2 \mathrm{~B}$. Then, the effective thermal conductivity and/or temperature distribution in specific examples are calculated to validate the proposed algorithm (Sec. 3A). For PZT thin films, the film-growing conditions, including the chemical composition [34], substrate-mismatch strain [35,36], film thickness [37-40], and film orientation [41-43], can be controlled to engineer the domain structure. A Piezoresponse Force Microscopy tip also can be used to modify the domain structure [44-49]. The effects of external conditions on domain structure as well as the effective thermal conductivity are studied in Sec. 3B. Before we conclude in Sec. 4, we provide a short discussion (Sec. 3C) on the possible challenges and future perspective in the theoretical prediction of domain wall-tuning of effective thermal conductivity in ferroelectric nanostructures.

\section{Method}

\section{A. Phase-field Model of a Ferroelectric Thin Film}

The phase-field model for ferroelectric thin films has been presented in numerous publications [50-54], and thus we focus on the phase-field description of inhomogeneous conductivity in a domain structure. For determining the domain-wall density in a given domain structure, we introduce a phase-field order parameter $\eta(\mathbf{x})$ to distinguish the domain-wall region and domain.

The polarization gradient energy density $f_{\text {grad }}(\mathbf{x})$ is used as a criterion to distinguish the domain interior and domain-wall region because the polarization gradient in the domain wall is much higher than in the domain. The general formulation of the gradient energy density in an anisotropic system can be expressed as 


$$
f_{\text {grad }}(\mathbf{x})=\frac{1}{2} \gamma_{i j k l} \frac{\partial P_{i}}{\partial x_{j}} \frac{\partial P_{k}}{\partial x_{l}}
$$

where $\gamma_{i j k l}$ is the gradient energy coefficient. For an isotropic system, Eq. (2-1) reduces to

$$
\begin{aligned}
f_{\text {grad }}(\mathbf{x})=\frac{1}{2} \gamma_{11} & {\left[\left(\frac{\partial P_{1}}{\partial x_{1}}\right)^{2}+\left(\frac{\partial P_{2}}{\partial x_{2}}\right)^{2}+\left(\frac{\partial P_{3}}{\partial x_{3}}\right)^{2}+\left(\frac{\partial P_{1}}{\partial x_{2}}\right)^{2}+\left(\frac{\partial P_{2}}{\partial x_{1}}\right)^{2}+\right.} \\
& \left.\left(\frac{\partial P_{1}}{\partial x_{3}}\right)^{2}+\left(\frac{\partial P_{3}}{\partial x_{1}}\right)^{2}+\left(\frac{\partial P_{2}}{\partial x_{3}}\right)^{2}+\left(\frac{\partial P_{3}}{\partial x_{2}}\right)^{2}\right]
\end{aligned}
$$

where $\gamma_{i j}$ is related to $\gamma_{i j k l}$ through the Voigt's notation and $\gamma_{12}=0, \gamma_{11}=2 \gamma_{44}$ in an isotropic system.

For $\eta(\mathbf{x})$, it can be written as follows by considering a diffuse interface

$$
\eta(\mathbf{x})=\frac{1}{2}\left\{1.0+\tanh \left[\beta\left(f_{\text {grad }}(\mathbf{x})-f_{c}\right)\right]\right\}
$$

where $\beta$ is a positive parameter controlling the width of the interface, $f_{\mathrm{c}}$ is a critical value of the polarization gradient energy density separating the bulk domain from the domain-wall region. Therefore, in the domain-wall region $\eta(\mathbf{x})=1$, and inside a domain $\eta(\mathbf{x})=0$. In this work, $\beta=500$ and $f_{\mathrm{c}}=0.15 \mathrm{Jm}^{-3}$ are used for $\mathrm{PbZr}_{1-x} \mathrm{Ti}_{x} \mathrm{O}_{3}$ films while assuming isotropic gradient energy coefficient.

The type of a domain wall that separates the two adjacent domains is determined using the product of their polarization vectors. For example, there are two domains, a and b, with respective polarization vectors $\mathbf{P}_{\mathrm{a}}$ and $\mathbf{P}_{\mathrm{b}}$ separated by a domain wall $L$. The type of domain wall $L$ is determined by $\theta(L)=\cos ^{-1}\left(\mathbf{P}_{\mathrm{a}} \square \mathbf{P}_{\mathrm{b}}\right) /\left(P_{\mathrm{a}} P_{\mathrm{b}}\right)$. For $\theta(L)=180^{\circ}, 90^{\circ}, 71^{\circ}$ and $109^{\circ}$, the corresponding domain walls are named $180^{\circ}, 90^{\circ}, 71^{\circ}$ and $109^{\circ}$ walls, respectively. 


\section{Acta Materialia}

The spatially dependent thermal conductivity in a ferroelectric thin film with domain structure then can be described as follows

$$
k_{i j}(\mathbf{x})=k_{i j}^{\mathrm{fs}-\text { wall }} \eta(\mathbf{x})+k_{i j}^{\text {domain }}(1-\eta(\mathbf{x}))
$$

where $k_{i j}^{\mathrm{fs}-w a l l}$ and $k_{i j}^{\text {domain }}$ represent the individual thermal conductivity of the ferroelastic domain wall and domain, respectively. For the $180^{\circ}$ ferroelectric domain wall, since the acoustic phonons that carry most of the thermal energy would have identical phonon dispersion spectra on either side of the domain wall, there would be no acoustic mismatch. Also, the amount of strain at a $180^{\circ}$ wall is very small, thus the $180^{\circ}$ ferroelectric domain wall is assumed to have the same thermal conductivity as the domain interior.

\section{B. Solution of the stationary heat conduction equation}

In order to obtain the effective thermal conductivity, it is necessary to solve the stationary heat conduction equation. Heat conduction in a material with inhomogeneous thermal conductivity distribution is governed by

$$
\frac{\partial}{\partial x_{i}}\left(k_{i j}(\mathbf{x}) \frac{\partial T(\mathbf{x})}{\partial x_{j}}\right)+q(\mathbf{x})=\rho c_{p} \frac{\partial T(\mathbf{x})}{\partial t}
$$

where $k_{i j}(\mathbf{x})$ is the spatial-dependent thermal conductivity tensor, $T(\mathbf{x})$ is the temperature distribution, $\rho, c_{p}$, and $q(\mathbf{x})$ are the mass density, specific heat capacity, and the internal heat source of the material, respectively. Under stationary conditions, which means sufficient time has passed such that the thermal field, $T(\mathbf{x})$, is no longer evolving with time, Eq. (2-5) reduces to

$$
\frac{\partial}{\partial x_{i}}\left(k_{i j}(\mathbf{x}) \frac{\partial T(\mathbf{x})}{\partial x_{j}}\right)+q(\mathbf{x})=0
$$




\section{Acta Materialia}

We consider a material system in which the thermal conductivity is periodic on the boundary and the thermal conductivity can be written as

$$
k_{i j}(\mathbf{x})=k_{i j}^{0}+\Delta k_{i j}(\mathbf{x})
$$

where $k_{i j}^{0}$ and $\Delta k_{i j}(\mathbf{x})$ represent the homogeneous and periodically inhomogeneous parts of the thermal conductivity, respectively. The stationary distribution of temperature depends on the boundary condition, and here we consider a homogeneous driving force for heat conduction in the material, i.e.,

$$
\frac{\partial T(\mathbf{x})}{\partial x_{j}}=\frac{\partial T^{\text {linear }}(\mathbf{x})}{\partial x_{j}}+\frac{\partial u(\mathbf{x})}{\partial x_{j}}=f_{j}+\frac{\partial u(\mathbf{x})}{\partial x_{j}}
$$

where $T^{\text {linear }}(\mathbf{x})$ represents the linear part of the temperature profile, and $u(\mathbf{x})$ represents the nonlinear part of the temperature profile that originates from the inhomogeneous distribution of the thermal conductivity and has a periodic distribution. Here we use $f_{j}$ to represent $\partial T^{\text {linear }}(\mathbf{x}) / \partial x_{j}$ for simplification. Combining Eq. (2-7) and (2-8), Eq. (2-6) can be written as

$$
\frac{\partial}{\partial x_{i}}\left[\left(k_{i j}^{0}+\Delta k_{i j}(\mathbf{x})\right)\left(f_{j}+\frac{\partial u(\mathbf{x})}{\partial x_{j}}\right)\right]+q(\mathbf{x})=0 .
$$

Rearranging Eq. (2-9) we get

$$
k_{i j}^{0} \frac{\partial^{2} u(\mathbf{x})}{\partial x_{i} \partial x_{j}}=-\frac{\partial}{\partial x_{i}}\left(\Delta k_{i j}(\mathbf{x})\left(f_{j}+\frac{\partial u(\mathbf{x})}{\partial x_{j}}\right)\right)-q(\mathbf{x})
$$

Zeroth-order Solution: Assuming the thermal conductivity to be homogeneous so that $\Delta k_{i j}(\mathbf{x})=0$, Eq. (2-10) reduces to 


$$
k_{i j}^{0} \frac{\partial^{2} u^{0}(\mathbf{x})}{\partial x_{i} \partial x_{j}}=-q(\mathbf{x})
$$

By performing a Fourier transformation on both sides of Eq. (2-11) we have

$$
-k_{i j}^{0} \xi_{i} \xi_{j} u^{0}(\xi)=-q(\xi)
$$

where $u^{0}(\xi)$ and $q(\xi)$ are the Fourier transforms of $u^{0}(\mathbf{x})$ and $q(\mathbf{x}), u^{0}(\xi)=\int_{V} u^{0}(\mathbf{x}) e^{-I \xi \cdot \mathbf{x}} d^{3} x$, $q(\xi)=\int_{V} q(\mathbf{x}) e^{-r \xi \cdot x} d^{3} x$, respectively, $\xi_{j}$ is the $j$ th component of the reciprocal lattice vector, $\xi$, and $I$ is the imaginary unit. From Eq. (2-12), we have the solution for $u^{0}(\xi)$,

$$
u^{0}(\xi)=G(\xi) q(\xi)
$$

where $G^{-1}(\xi)=k_{i j}^{0} \xi_{i} \xi_{j}$. By performing inverse Fourier transforms on both sides of Eq. (2-13), the zeroth-order approximation of the nonlinear part $u^{0}(\mathbf{x})$ of the temperature profile is

$$
u^{0}(\mathbf{x})=\int \frac{d^{3} \xi}{(2 \pi)^{3}} G(\xi) q(\xi) e^{I \xi \cdot \mathbf{x}}
$$

First-order Solution: Substituting the zeroth-order solution of the nonlinear part of the temperature profile (Eq. (2-14)) into the iterative Eq. (2-10), the first-order solution of $u(\mathbf{x})$ can be obtained from

$$
k_{i j}^{0} \frac{\partial^{2} u^{1}(\mathbf{x})}{\partial x_{i} \partial x_{j}}=-\frac{\partial}{\partial x_{i}}\left[\Delta k_{i j}(\mathbf{x})\left(f_{j}+\frac{\partial u^{0}(\mathbf{x})}{\partial x_{j}}\right)\right]-q(\mathbf{x}) .
$$

Similarly, by performing a Fourier transformation on both sides of Eq. (2-15) we have 


$$
u^{1}(\xi)=I G \xi_{i}\left\{\Delta k_{i j}(\mathbf{x})\left(f_{j}+\frac{\partial u^{0}(\mathbf{x})}{\partial x_{j}}\right)\right\}_{\xi}+q(\xi)
$$

where $\left\{\Delta k_{i j}(\mathbf{x})\left(f_{j}+\frac{\partial u^{0}(\mathbf{x})}{\partial x_{j}}\right)\right\}_{\xi}$ indicates the Fourier transform of $\Delta k_{i j}(\mathbf{x})\left(f_{j}+\frac{\partial u^{0}(\mathbf{x})}{\partial x_{j}}\right)$. As a result, the first-order solution of the nonlinear part of the temperature profile can be obtained using

$$
u^{1}(\mathbf{x})=\int \frac{d^{3} \xi}{(2 \pi)^{3}} u^{1}(\xi) e^{I \xi \cdot x}
$$

Higher-order Solution: The higher-order solutions for $u(\mathbf{x})$ are derived in a similar manner as the first-order solution,

$$
k_{i j}^{0} \frac{\partial^{2} u^{n}(\mathbf{x})}{\partial x_{i} \partial x_{j}}=-\frac{\partial}{\partial x_{i}}\left[\Delta k_{i j}(\mathbf{x})\left(f_{j}+\frac{\partial u^{n-1}(\mathbf{x})}{\partial x_{j}}\right)\right]-q(\mathbf{x})
$$

Similar to $u^{1}(\mathbf{x})$, the solution for $u^{n}(\mathbf{x})$ is solved using Fourier transforms

$$
u^{n}(\xi)=I G \xi_{i}\left\{\Delta k_{i j}(\mathbf{x})\left(f_{j}+\frac{\partial u^{n-1}(\mathbf{x})}{\partial x_{j}}\right)\right\}_{\xi}+q(\xi)
$$

and the $n$ th-order solution of the nonlinear part of the temperature can be obtained by

$$
u^{n}(\mathbf{x})=\int \frac{d^{3} \xi}{(2 \pi)^{3}} u^{n}(\xi) e^{I \xi \cdot \mathbf{x}}
$$

As for the total temperature distribution $T(\mathbf{x})$, it can be calculated from 


$$
T(\mathbf{x})=u^{n}(\mathbf{x})+\int f_{j} d x_{j}
$$

while taking into account the boundary conditions.

According to the Fourier's law, the heat flux density that flows through a unit area per unit time is equal to

$$
J_{i}=-k_{i j}(\mathbf{x}) \partial T(\mathbf{x}) / \partial x_{j}
$$

The effective thermal conductivity tensor $k_{i j}^{\text {eff }}$ of the material can be determined from Eq. (2-22) by solving

$$
\left\langle J_{i}\right\rangle=-k_{i j}^{\mathrm{eff}}\left\langle\partial T(\mathbf{x}) / \partial x_{j}\right\rangle
$$

where \langle\rangle represents the average property per volume.

\section{Results and Discussions}

\section{A. Testing Examples}

\section{A1. Parallel slabs}

As the first test example, we calculate the effective thermal conductivity for a simple microstructure composed of parallel slabs of A and B with isotropic, but different, thermal conductivities, $k_{\mathrm{A}}^{\text {iso }}$ and $k_{\mathrm{B}}^{\text {iso }}$, as exhibited in Fig. 1a. In this case, the effective thermal conductivity can be expressed analytically so as to be compared with the numerical results from the SIP method shown in Sec. 2B. Assuming that the heat flux is along the 22 direction, which is parallel to the plane of the slabs, the arrangement is equivalent to a parallel electrical circuit wherein each slab has the same temperature gradient but different heat flux. As a result, the effective thermal conductivity component along the 22 direction is given by $k_{22}^{\text {eff }}=k_{\mathrm{A}}^{\text {iso }} V_{\mathrm{A}}+k_{\mathrm{B}}^{\text {iso }} V_{\mathrm{B}}$, 


\section{Acta Materialia}

where $V_{\mathrm{A}}$ and $V_{\mathrm{B}}$ are the volume fractions of component $\mathrm{A}$ and $\mathrm{B}$, respectively. Similarly, for heat flux along the 11 direction, which is perpendicular to the plane of the slabs (see Fig. 1a), it is equivalent to a series electrical circuits wherein each slab has the same heat flux but different temperature gradients. As a result, the effective thermal conductivity component along the 11 direction is given by $k_{11}^{\text {eff }}=k_{\mathrm{A}}^{\text {iso }} k_{\mathrm{B}}^{\text {iso }} /\left(k_{\mathrm{A}}^{\text {iso }} V_{\mathrm{B}}+k_{\mathrm{B}}^{\text {iso }} V_{\mathrm{A}}\right)$. Fig. 1b shows the comparison of numerical and theoretical $\mathbf{k}^{\text {eff }}$ as a function of volume fraction for these two parallel slab microstructures. As it can be seen from the comparison, the effective thermal conductivity calculated from the SIP method agrees well with those of theoretical solutions, thus validating the proposed spectral method. Additionally, although the thermal conductivity of each composition is isotropic, the effective thermal conductivity is anisotropic due to the anisotropic microstructure.

\section{A2. Square matrix with a circular inclusion}

In order to further test the accuracy of SIP method on solving the stationary heat conduction equation with inhomogeneous thermal conductivity distribution, we consider a two-phase microstructure composed of a square matrix and a circular inclusion, as shown in Fig. 2a. These two phases are assumed to have isotropic but different thermal conductivities. The boundary conditions are set as following: temperatures on the left and right boundaries are fixed at $300 \mathrm{~K}$ and $500 \mathrm{~K}$, and the bottom and top boundaries are adiabatic, as illustrated in Fig. 2a. The ratio of $k^{\mathrm{p}}$ to $k^{\mathrm{m}}$ has been set to a value of 1:400 and the ratio of the length of the matrix (denoted by $d$ ) to the radius of the inclusion (denoted by $r$ ) has been set to a value of 10:1. Fig. $2 \mathrm{~b}$ gives the temperature distribution at the middle cross section of the composite for these conditions (see the red dashed line in Fig. 2a solved from the proposed SIP method, compared with the results obtained from the finite element method (FEM) using COMSOL software. It can be seen that the two solutions are nearly identical, further validating the proposed spectral method. 


\section{Acta Materialia}

Fig. $2 \mathrm{c}$ and $2 \mathrm{~d}$ show the numerically calculated effective thermal conductivity as a function of the volume fraction of the inclusion $V_{\mathrm{p}}$ and $k^{\mathrm{p}} / k^{\mathrm{m}}$, respectively, compared with the results obtained from the Maxwell-Garnett effective medium model [55,56], where the size or the thermal conductivity mismatch between the inclusion and the matrix has been varied. It can be seen that the effective thermal conductivities obtained from these two methods agree well. The advantage of the SIP method is that it is able to compute not only the effective thermal conductivity but also the temperature distribution.

\section{A3. Experimental effective thermal conductivity of $\mathrm{BiFeO}_{3}$ films}

The domain structure in epitaxial $\mathrm{BiFeO}_{3}$ thin films can be engineered utilizing substrate vicinality to constrain growth and alter the number of domain variants [57-59]. Fig. 3a and $3 b$ show the experimentally measured and simulated out-of-plane effective thermal conductivities at different temperatures for $30 \mathrm{~nm}$ thick (001)-oriented $\mathrm{BiFeO}_{3}$ thin films with 2-variant and 4variant domain structures, respectively [20]. Only $71^{\circ}$ domain walls exist in these two domain structures. In the simulation, the thermal conductivity of the domain was taken from the experimental measurements on the sample without domain walls, and the thermal conductivity of the wall was obtained using the experimentally derived Kapitza conductance of these $71^{\circ}$ domain walls and multiplying by an estimated domain wall thickness $(2.4 \mathrm{~nm}$, about 6 unit cells $[60,61])$, as shown in Fig. 3c. At a temperature of $295 \mathrm{~K}$, the domain has a thermal conductivity of $k^{\text {domain }}$ $=2.16 \mathrm{Wm}^{-1} \mathrm{~K}^{-1}$, while the ferroelastic domain wall has a value of $k^{\mathrm{fs}-\text { wall }}=0.244 \mathrm{Wm}^{-1} \mathrm{~K}^{-1}$; in other words, the wall has a much higher thermal resistance than the domain interior. For the film with the 2-variant domain structures having a domain-wall volumetric density of $3.3 \%$, the experimental effective thermal conductivity of the film is $1.68 \pm 0.00 \mathrm{Wm}^{-1} \mathrm{~K}^{-1}$, compared with $1.602 \mathrm{Wm}^{-1} \mathrm{~K}^{-1}$ from simulation. While for the film with 4-variant domain structures and a 


\section{Acta Materialia}

domain-wall volumetric density of $4.8 \%$, the experimental and simulated effective thermal conductivities are $1.50 \pm 0.08$ and $1.552 \mathrm{Wm}^{-1} \mathrm{~K}^{-1}$, respectively. Agreement between the experimental and simulated values validates the use of a separate region with finite thickness to define the thermal properties of domain walls in these simulations.

\section{B. Tunable thermal conductivity in PZT films}

Based on the above tests on the proposed SIP algorithm for obtaining the effective thermal conductivity in different structures, we can see that the SIP method is an efficient tool for interpreting the relationship between the effective property and microstructure. Hereafter we use it to study the tunable effective thermal conductivity in PZT films by engineering the ferroelectric domain structure. Due to the lack of experimental data or first-principle calculation on the ferroelastic domain-wall thermal conductivity, or Kaptiza conductance, for PZT, here a ratio of $k^{\text {domain }} / k^{\text {fs-wall }}=10$ at room temperature (a ratio of $\approx 9$ was used for the $(001)$-oriented $\mathrm{BiFeO}_{3}$ film from experiment) is used to evaluate the effective thermal conductivity with different domain structures under various conditions. Although different types of ferroelastic domain walls and different compositions of PZT should have different thermal conductivities, here we assume the same thermal conductivity for all types of ferroelastic domain walls in PZT due to the lack of experimental data. As noted above, the ferroelectric-only $180^{\circ}$ domain walls are assumed to have the same thermal conductivity as the domain interior. As a result, the effective thermal conductivity will be mainly determined by the density and arrangement of the ferroelastic domain walls.

\section{B1. Chemical Composition Effect}

Here when referring the chemical composition effect, we focus on its effect on the domain structures and, further, the effective thermal conductivity of PZT thin films clamped by a 


\section{Acta Materialia}

substrate with a zero mismatch strain. We will not discuss the effect of alloy scattering due to the intermixing of $\mathrm{Ti}$ and $\mathrm{Zr}$ on the $\mathrm{B}$-site of $\mathrm{ABO}_{3}$ perovskite. As the Ti composition increases, the ferroelectric state transits from rhombohedral to tetragonal. Fig. 4a shows the domain-wall density and type as function of Ti composition. It can be seen that for compositions with $x_{\mathrm{Ti}} \leq 0.5$, the total domain wall density is small and decreases slightly with composition. However, for $x_{\mathrm{Ti}}>$ 0.5, the domain-wall density increases significantly with Ti composition. As a result, the effective thermal conductivity, particularly the out-of-plane component, decreases generally with increasing Ti composition, as illustrated in Fig. 4b. When $x_{\mathrm{Ti}}=1.0$, i.e., for a $\mathrm{PbTiO}_{3}$ film, the effective thermal conductivity components along the 11,22 , and 33 directions are $67 \%, 78 \%$, and $82 \%$ of the single domain value, respectively. Such a decrease and anisotropy in the effective thermal conductivity when $x_{\mathrm{Ti}}>0.5$ can be understood from the domain structure morphologies, as shown in Fig. 4c. When $x_{\mathrm{Ti}} \leq 0.5$, the dominant ferroelectric phase is rhombohedral with low population of domain walls, yet when $x_{\mathrm{Ti}}>0.5$ the dominant ferroelectric phase is tetragonal with dense populations of $90^{\circ}$ ferroelastic domain walls. The large increase of $90^{\circ}$ ferroelastic domain walls, oriented at about $45^{\circ}$ with respect to the heat flux direction, can significantly scatter phonons and resist the thermal transport.

\section{B2. Misfit Strain Effect}

Fig. 5a shows the strain-domain phase diagram for $\mathrm{PbZr}_{0.2} \mathrm{Ti}_{0.8} \mathrm{O}_{3}$ films. When the substrateinduced strain is compressive and larger than $-0.4 \%$, most of the domains in the film are out-ofplane $c$ domains (see Fig. 5a) and most of the domain walls are $180^{\circ}$-type (see Fig. 5b) nonferroelastic walls. When the substrate-induced strain is tensile and larger than $1.0 \%$, most of the domains in the film are in-plane $a a$ twinning domains, as shown from the simulated domain structures in the inset of Fig. 5a. This ordered $a a$ twinning domain structure is very similar to the 


\section{Acta Materialia}

experimental image for the $\mathrm{PbTiO}_{3}$ thin film grown on a $\mathrm{KTaO}_{3}$ substrate [62]. Most of the domain walls in $a a$ twinning domain structures are $90^{\circ}$-type (see Fig. 5b) ferroelastic walls. When the substrate-induced strain is moderate and between $-0.4 \%$ and $1.0 \%$, out-of-plane $c$ domains and in-plane $a$ domains coexist in the film. When the misfit strain is tensile, the dominant domain walls are $90^{\circ}$ type. In general, the total domain wall density increases with the magnitude of misfit strain, as it can be seen from Fig. $5 b$.

The effective thermal conductivity as a function of misfit strain is shown in Fig. 5c. Generally, with the misfit strain changing from compressive to tensile, the effective thermal conductivity decreases due to the increase of the ferroelastic $90^{\circ}$-type domain-wall density. In most cases the out-of-plane thermal conductivity is different from the in-plane counterpart, exhibiting a strong anisotropy due to the dimensions of the film. The mechanism that strain can increase the domain wall density and further decrease the effective thermal conductivity can be understood from the strain effect on the domain size and, consequently, on the number of ferroelastic domain walls present. With the magnitude of the misfit strain becoming larger, the domain size becomes smaller to introduce more inhomogeneous strain to release the elastic energy, giving rise to high densities of domain walls [63]. It is worth noting that the effective thermal conductivity decreases to a local minimum with the misfit strain varying from compressive to zero (see Fig. $5 \mathrm{c}$ ), and then increases until the misfit strain becomes $0.6 \%$. This non-monotonic variation of the effective thermal conductivity with strain corresponds to the $90^{\circ}$ type ferroelastic domain wall change (see Fig. 5b), which is caused by the transition of ferroelastic walls from the $a c$ walls to $a a$ walls.

\section{B3. Film Thickness Effect}




\section{Acta Materialia}

For $\mathrm{PbZr}_{0.2} \mathrm{Ti}_{0.8} \mathrm{O}_{3}$ films epitaxially grown on $\mathrm{SrTiO}_{3}$ substrates, the ideal strain due to lattice mismatch reaches up to $-2.38 \%$, which can generate significant internal stress. With film thickness increasing, such internal stress will be relaxed through the formation of either dislocations or ferroelastic twins. Fig. 6a shows the effective misfit strain as function of film thickness obtained from the People-Bean (PB) model $[40,64]$ which predicts a critical thickness of $8 \mathrm{~nm}$ for dislocation formation. According to the PB model, when the film is thinner than $8 \mathrm{~nm}$, the thin film can be regarded as fully constrained by the substrate, giving rise to an ideal misfit strain of $-2.38 \%$. With the film thickness increasing to $100 \mathrm{~nm}$, the effective misfit strain decreases to $-0.21 \%$. Therefore, the domain structures will be different for films with different thickness although the chemical composition and substrate are the same. Fig. 6b shows the domain structures for films with thickness of $10 \mathrm{~nm}, 40 \mathrm{~nm}$, and $100 \mathrm{~nm}$, respectively. For the $10 \mathrm{~nm}$-thick and $40 \mathrm{~nm}$-thick films, all domains are out-of-plane $c$ domains separated by $180^{\circ}$ domain walls, which are non-ferroelastic, however the domain size of these two films are very different. The thinner the film, the smaller the domain size will be because of the stronger misfit strain. For the $100 \mathrm{~nm}$-thick film, the out-of-plane $c$ domains and in-plane $a$ domains coexist due to the moderate misfit strain. The effects of film thickness on the domain structures are essentially same as the misfit strain effect, as discussed in Sec. 3B2.

Fig. 6c shows the domain wall density as function of film thickness. For films with thicknesses smaller than $40 \mathrm{~nm}$, there are only $180^{\circ}$ type ferroelectric domain walls and no ferroelastic domain walls. When the film thickness is thicker than $40 \mathrm{~nm}$, the $90^{\circ}$ ferroelastic domain walls begin to emerge and coexist with the $180^{\circ}$ ferroelectric domain walls. Fig. $6 \mathrm{~d}$ shows the effective thermal conductivity for films with different thicknesses. With film thickness increasing, the effective thermal conductivity decreases. It is worth noting here that all data 


\section{Acta Materialia}

points for the domain wall density and effective thermal conductivity are obtained by averaging ten different quasi-equilibrium domain structures under a specific condition, and the bar of each data point shows the corresponding standard deviation. For films with thickness smaller than $50 \mathrm{~nm}$, which are severely constrained by the substrate according to the PB model, there are only out-of-plane c domains and thus no ferroelastic domain walls, so the effective thermal conductivity is nearly the same as inside the domains, as exhibited in Fig. 6d.

We note that the film thickness dependent thermal conductivity results presented in this section do not account for size effects due to film boundary scattering $[65,66]$. Clearly, for thin film systems, phonon-boundary scattering can lead to pronounced size effects in the thermal conductivity depending on the phonon mean free paths relative to the film thickness. However, the goal of this work is to isolate the role of domain boundary resistance on the thermal conductivity of thin films. In experimental data, multiple competing effects will be occurring in addition to this phonon-domain scattering effect. These results presented in this section can begin to help to deconvolve these mechanisms.

\section{B4. Film Orientation Effect}

$\{001\}_{p^{-}},\{011\}_{p^{-}}$, and $\{111\}_{p^{-}}$oriented (where the $p$ subscript denotes pseudocubic indices) $\mathrm{PbZr}_{0.2} \mathrm{Ti}_{0.8} \mathrm{O}_{3}$ films can be obtained by growing the films on $\mathrm{SrTiO}_{3}$ substrates with corresponding orientations [43]. Fig. 7a shows the ferroelastic domain wall density as well as the domain structures for the $\{001\}_{p^{-}},\{011\}_{p^{-}}$, and $\{111\}_{p^{-}}$oriented films in the as-grown state. The $\{001\}_{p^{-}}$oriented film shows the highest ferroelastic domain wall density, followed by the $\{111\}_{p^{-}}$ oriented film, and the $\{011\}_{p}$-oriented film last. The $\{001\}_{p}$-oriented film shows the lowest thermal conductivity value for the in-plane components. The out-of-plane component of the effective thermal conductivity for $\{001\}_{p^{-}}$and $\{111\}_{p^{-}}$oriented films are similar. The lowest 


\section{Acta Materialia}

ferroelastic domain wall density for the $\{011\}_{p}$-oriented film results in the highest out-of-plane component of effective thermal conductivity.

\section{B5. Piezoresponse Force Microscopy Tip Effect}

Piezoresponse force microscopy (PFM) has been used extensively to image and control the domain structures of ferroelectric thin films $[44-49,67,68]$. When a PFM tip bias is applied on a ferroelectric thin film, the polarization can be locally or even collectively switched due to the strong coupling between the polarization and electromechanical response. Fig. 8a shows the domain structure evolution for a $\mathrm{PbZr}_{0.2} \mathrm{Ti}_{0.8} \mathrm{O}_{3}$ thin film (20 nm thickness) under zero mismatch strain and with $a_{1} c^{+}$tetragonal domain variants. Before applying a PFM tip bias, this simple $a_{1} c^{+}$ domain structure is stable and only has $90^{\circ}$-type domain walls with a volume fraction of $2.7 \%$ (see the domain structures and domain-wall density at a time step of 30000 in Fig. 8a). Upon applying a PFM tip with $10 \mathrm{~nm}$ radius and $5 \mathrm{~V}$ bias on top of the film, $c^{-}$domain and $a_{2}$ domains are generated, giving rise to an increase to $9.4 \%$ in the total domain-wall volumetric density (see the domain structures and domain-wall density at a time step of 60000 in Fig. 8a). Most of this induced domain-wall density increase can be retained after the removal of the PFM tip bias (see the domain structures and domain-wall density at time steps of 90000 in Fig. 8a). This PFM tip bias-induced local $90^{\circ}$ and $180^{\circ}$ domain switching in tetragonal PZT films can occur in favor of releasing the elastic energy and electric energy, respectively, as analyzed previously in the work of Hong et al. [49].

Accompanying the increase in domain-wall density induced by PFM tip bias, the effective thermal conductivity is modified correspondingly. As shown in Fig. 8b, prior to the application of PFM tip bias, the three components of the effective thermal conductivity are $71.1 \%, 97.2 \%$, and $95.1 \%$ of the single domain value (see $k$ values at a time step of 30000 in Fig. 


\section{Acta Materialia}

8b), respectively. Under PFM tip bias, the three components of the effective thermal conductivity are changed to $90.9 \%, 84.2 \%$, and $88.9 \%$ of the single domain value, respectively (see $k$ values at a time step of 60000 in Fig. 8b). After the removal of the PFM tip bias, the three components of the effective thermal conductivity are retained at $88.3 \%, 84.6 \%$, and $88.6 \%$ of the domain value, respectively (see $k$ values at a time step of 90000 in Fig. 8b).

\section{Discussion}

From above simulations we can see that the effective thermal conductivity in ferroelectric thin films can be tuned via domain structure engineering by a number of means. However, we have to acknowledge that the prediction from present simulation needs further verification due to the lack of experimental data for the input parameters such as the thermal conductivity tensors for different types of domains and domain walls. Presently, measurement of thermal properties of a single domain wall presents an experimental challenge. Additionally, the coupling mechanisms between phonons, polarization, and strain at domain walls remain unclear. However, a combination of the density function theory (DFT) calculation and molecular dynamics (MD) simulation may be a feasible way to obtain the thermal conductivity tensors at the domain and domain wall. With the knowledge of the thermal conductivity tensors of the domain (bulk) and domain walls (interfaces), the present proposed spectral iterative perturbation algorithm combined with the phase-field model of ferroelectric films provides an efficient means to predict the effective thermal conductivity as a function of domain structure (microstructures).

\section{Conclusions}

The heat conduction equation in materials with inhomogeneous thermal conductivity distribution is solved using a spectral iterative perturbation method to obtain the effective thermal conductivity. Based on this method, the effective thermal conductivities for engineered $\{001\}_{p^{-}}$ 


\section{Acta Materialia}

$\mathrm{BiFeO}_{3}$ thin films are calculated and the obtained results quantitatively agree with the experimentally measured values. By combining this SIP method with the phase-field model of ferroelectric thin films, the effective thermal conductivity as function of domain structure is studied. It is found that for $\mathrm{PbZr}_{1-x} \mathrm{Ti}_{x} \mathrm{O}_{3}$ films, many factors including the chemical composition, misfit strain, film thickness, film orientation, and piezoresponse force microscopy tip can be used to engineer the domains structure and tune the effective thermal conductivity. For chemical composition, increasing $\mathrm{PbTiO}_{3}$ composition results in increased ferroelastic domain wall concentrations and contributes to decreases in the effective thermal conductivity. For misfit strain, enhancing the tensile misfit strain increases the ferroelastic domain wall density and decreases the effective thermal conductivity. For film thickness, it affects the effective thermal conductivities according to the manner similar to the misfit strain. For film orientation, $\{001\}_{p^{-}}$ oriented films show the highest ferroelastic domain wall density and further the lowest effective thermal conductivity. Piezoresponse force microscopy tip bias can also be used to alter the domain structure and the effective thermal conductivity. The present simulation provides not only an efficient way to obtain the effective thermal conductivity as function of microstructure but also an insight on how to tune the effective thermal conductivity of ferroelectric thin films via domain structure engineering.

\section{Acknowledgement}

This work was funded, in-part, by the Laboratory Directed Research and Development Program at Sandia National Laboratories, a multi-program laboratory managed and operated by Sandia Corporation, a wholly owned subsidiary of Lockheed Martin Corporation, for the U.S. Department of Energy's National Nuclear Security Administration under contract DE-AC0494AL85000, and partially supported by Air Force Office of Scientific Research (AFOSR) under 


\section{Acta Materialia}

grant number FA9550-14-1-0264. PEH appreciates support from the Air Force Office of

Scientific Research (FA9550-15-1-0079). The computations were performed using the Cyberstar

cluster at the Pennsylvania State University, funded by the National Science Foundation through

Grant OCI-0821527, and the National Energy Research Scientific Computing Center, funded by

the Office of Science of the U.S. Department of Energy through Grant DE-AC02-05CH11231.

[1] P. E. Hopkins, C. M. Reinke, M. F. Su, R. H. Olsson III, E. A. Shaner, Z. C. Leseman, J. R. Serrano, L. M. Phinney, I. E. Kady, Reduction in the Thermal Conductivity of Single Crystalline Silicon by Phononic Crystal Patterning, Nano Lett. 11 (2011) 107-112.

[2] M. Zebarjadi, K. Esfarjani, M. S. Dresselhaus, Z. F. Ren, G. Chen, Perspectives on thermoelectrics: from fundamentals to device applications, Energy Environ. Sci. 2 (2009) 466-479.

[3 ] S. M. Lee, D. G. Cahill, R. Venkatasubramanian, Thermal conductivity of Si-Ge superlattices, Appl. Phys. Lett. 70 (22) (1997) 2957-2959.

[4] G. Chen, Thermal conductivity and ballistic-phonon transport in the cross-plane direction of superlattices, Phys. Rev. B 57 (23) (1998) 14958-14973.

[5] M. N. Luckyanova, J. Garg, K. Esfarjani, A. Jandl, M. T. Bulsara, A. J. Schmidt, A. J. Minnich, S. Chen, M. S. Dresselhaus, Z. Ren, E. A. Fitzgerald, and G. Chen, Coherent phonon heat conduction in superlattices. Science, 338 (6109) (2012) 936-939, 2012.

[6] J. Ravichandran, A. K. Yadav, R. Cheaito, P. B. Rossen, A. Soukiassian, S. J. Suresha, J. C. Duda, B. M. Foley, C.-H. Lee, Y. Zhu, A. W. Lichtenberger, J. E. Moore, D. A. Muller, D. G. Schlom, P. E. Hopkins, A. Majumdar, R. Ramesh, and M. A. Zurbuchen, Crossover from incoherent to coherent phonon scattering in epitaxial oxide superlattices, Nature Materials 13 (2014) 168-172.

[7] A. I. Hochbaum, R. Chen, R. D. Delgado, W. Liang, E. C. Garnett, M. Najarian, A. Majumdar and P. Yang, Enhanced thermoelectric performance of rough silicon nanowires, Nature 451 (2008) 163-167.

[8] A. I. Boukai, Y. Bunimovich, J. Tahir-Kheli, J.-K. Yu, W. A. Goddard Iii and J. R. Heath, Silicon nanowires as efficient thermoelectric materials, Nature 451 (2008) 168-171.

[9] T. C. Harman, P. J. Taylor, M. P. Walsh and B. E. LaForge, Quantum Dot Superlattice Thermoelectric Materials and Devices, Science 297 (5590) (2002) 2229-2232.

[10] P. E. Hopkins, J. C. Duda, C. W. Petz, and J. A. Floro, Controlling thermal conductance through quantum dot roughening at interfaces, Phys. Rev. B, 84 (2011) 035438.

[11] M. L. Lee and R. Venkatasubramanian, Effect of nanodot areal density and period on thermal conduc- tivity in sige/si nanodot superlattices, Appl. Phys. Lett. 92 (2008) 053112.

[12] G. Pernot, M. Stoffel, I. Savic, F. Pezzoli, P. Chen, G. Savelli, A. Jacquot, J. Schumann, U. Denker, I. Monch, C. Deneke, O. G. Schmidt, J. M. Rampnoux, S. Wang, M. Plissonnier, A. Rastelli, S. Dilhaire, and N. Mingo, Precise control of thermal conductivity at the nanoscale through individual phonon- scattering barriers, Nat. Mater. 9 (2010) 491-495. 
[13] C. W. Nan, R. Birringer, D. R. Clarke, H. Gleiter, Effective thermal conductivity of particulate composites with interfacial thermal resistance, J. Appl. Phys. 81 (10) (1997) 6992-6999.

[14] C. W. Nan, G. Liu, Y. H. Lin, M. Li, Interface effect on thermal conductivity of carbon nanotube composites, Appl. Phys. Lett. 85 (16) (2004) 3549-3551.

[15] B. Poudel, Q. Hao, Y. Ma, Y. Lan, A. Minnich, B. Yu, X. Yan, D. Wang, A. Muto, D. Vashaee, X. Chen, J. Liu, M. S. Dresselhaus, G. Chen and Z. Ren, High-Thermoelectric Performance of Nanostructured Bismuth Antimony Telluride Bulk Alloys, Science 320 (5876) (2008) 634-638.

[16] X. W. Wang, H. Lee, Y. C. Lan, G. H. Zhu, G. Joshi, D. Z. Wang, J. Yang, A. J. Muto, M. Y. Tang, J. Klatsky, S. Song, M. S. Dresselhaus, G. Chen, Z. Ren, Enhanced thermoelectric figure of merit in nanostructured n-type silicon germanium bulk alloy, Appl. Phys. Lett. 93 (19) (2008) 193121.

[17] G. Joshi, H. Lee, Y. Lan, X. Wang, G. Zhu, D. Wang, R. W. Gould, D. C. Cuff, M. Y. Tang, M. S. Dresselhaus, G. Chen, Z. Ren, Enhanced Thermoelectric Figure-of-Merit in Nanostructured p-type Silicon Germanium Bulk Alloys, Nano Lett. 8 (12) (2008) 4670-4674.

[18] A. J. H. Mante, J. Volger, Phonon transport in barium titanate, Physica 52 (4) (1971) 577604.

[19] M. A. Weilert, M. E. Msall, A. C. Anderson, and J. P. Wolfe, Phonon Scattering from Ferroelectric Domain Walls: Phonon Imaging in KDP, Phys. Rev. Lett. 71 (5) (1993) 735 738.

[20] P. E. Hopkins, C. Adamo, L. Ye, B. D. Huey, S. R. Lee, D. G. Schlom, J. F. Ihlefeld, Effects of coherent ferroelastic domain walls on the thermal conductivity and Kapitza conductance in bismuth ferrite, Appl. Phys. Lett. 102 (12) (2013) 121903.

[21] J. F. Ihlefeld, B. M. Foley, D. A. Scrymgeour, J. R. Michael, B. B. McKenzie, D. L. Medlin, M. Wallace, S. T. McKinstry, P. E. Hopkins, Room-Temperature Voltage Tunable Phonon Thermal Conductivity via Reconfigurable Interfaces in Ferroelectric Thin Films, Nano Lett. 15 (3) (2015) 1791-1795.

[22] W. Pompe, X. Gong, Z. Suo, J. S. Speck, Elastic energy release due to domain formation in the strained epitaxy of ferroelectric and ferroelastic films, J. Appl. Phys. 74 (10) (1993) 6012-6019.

[23] M. Tachibana, T. Kolodiazhnyi, E. T. Muromachi, Appl. Phys. Lett. 93 (9) (2008) 092902.

[24] B. M. Foley, H. J. Brown-Shaklee, J. C. Duda, R. Cheaito, B. J. Gibbons, D. Medlin, J. F. Ihlefeld, P. E. Hopkins, Appl. Phys. Lett. 101 (23) (2012) 231908.

[25] J. F. Bisson, H. Yagi, T. Yanagitani, A. Kaminskii, Y. N. Barabanenkov, K. I. Ueda, Influence of the Grain Boundaries on the Heat Transfer in Laser Ceramics, Opt. Rev. 14 (1) (2007) 1-13.

[26] B. F. Donovan, B. M. Foley, J. F. Ihlefeld, J. P. Maria, P. E. Hopkins, Spectral phonon scattering effects on the thermal conductivity of nano-grained barium titanate, Appl. Phys. Lett. 105 (8) (2014) 082907.

[27] Y. L. Li, S. Y. Hu, Z. K. Liu, L. Q. Chen, Phase-field model of domain structures in ferroelectric thin films, Appl. Phys. Lett. 78 (24) (2001) 3878-3880.

[28] Y. L. Li, S. Y. Hu, Z. K. Liu, L. Q. Chen, Effect of substrate constraint on the stability and evolution of ferroelectric domain structures in thin films, Acta Mater. 50 (2) (2002) 395-411. 
[29] Y. L. Li, S. Choudhury, Z. K. Liu, L. Q. Chen, Effect of External Mechanical Constraints on the Phase Diagram of Epitaxial $\mathrm{PbZr}_{1-x} \mathrm{Ti}_{x} \mathrm{O}_{3}$ Thin Films - Thermodynamic Calculations and Phase-Field Simulations, Appl. Phys. Lett. 83 (8) (2003) 1608-1610.

[30] S. Y. Hu, L. Q. Chen, A phase-field model for evolving microstructures with strong elastic inhomogeneity, Acta Mater. 49 (11) (2001) 1897-1890.

[31] J. J. Wang, S. Bhattacharyya, Q. Li, T. W. Heo, X. Q. Ma, L. Q. Chen, Elastic solutions with arbitrary elastic inhomogeneity and anisotropy, Philosophical Magazine Letters 92 (7) (2012) 327-335.

[32] J. J. Wang, X. Q. Ma, Q. Li, J. Britson, L. Q. Chen, Phase transitions and domain structures of ferroelectric nanoparticles: Phase field model incorporating strong elastic and dielectric inhomogeneity, Acta Mater. 61 (20) (2013) 7591-7603.

[33] J. J. Wang, Y. Song, X. Q. Ma, L. Q. Chen, C. W. Nan, Static magnetic solution in magnetic composites with arbitrary susceptibility inhomogeneity and anisotropy, J. Appl. Phys. 117 (4) (2015) 043907.

[34] K. S. Lee, J. H. Choi, J. Y. Lee, S. Baik, Domain formation in epitaxial $\mathrm{Pb}(\mathrm{Zr}, \mathrm{Ti}) \mathrm{O}_{3}$ thin films, J. Appl. Phys. 90 (8) (2001) 4095-4102.

[35] J. S. Speck, A. Seifert, W. Pompe, R. Ramesh, Domain configurations due to multiple misfit relaxation mechanisms in epitaxial ferroelectric thin films. II. Experimental verification and implications, J. Appl. Phys. 76 (1) (1994) 477-483.

[36] D. G. Schlom, L. Q. Chen, C. B. Eom, K. M. Rabe, S. K. Streiffer, J. M. Triscone, Strain Tuning of Ferroelectric Thin Films, Annu. Rev. Mater. Res. 37 (2007) 589-626

[37] D. D. Fong, G. B. Stephenson, S. K. Streiffer, J. A. Eastman, O. Auciello, P. H. Fuoss, C. Thompson, Science 304 (2004) 1650.

[38] T. Tybell, C. H. Ahn, J. M. Triscone, Ferroelectricity in thin perovskite films, Appl. Phys. Lett. 75 (6) (1999) 856.

[39] V. Nagarajan, J. Junquera, J. Q. He, C. L. Jia, R. Waser, K. Lee, Y. K. Kim, S. Baik, T. Zhao, R. Ramesh, Ph. Ghosez, K. M. Rabe, Scaling of structure and electrical properties in ultrathin epitaxial ferroelectric heterostructures, J. Appl. Phys. 100 (5) (2006) 051609.

[40] G. Sheng, J. M. Hu, J. X. Zhang, Y. L. Li, Z. K. Liu, L. Q. Chen, Phase-field simulations of thickness-dependent domain stability in $\mathrm{PbTiO}_{3}$ thin films, Acta Mater. 60 (8) (2012) 32963301.

[41] R. J. Xu, J. L. Zhang, Z. H. Chen, L. W. Martin, Orientation-dependent structural phase diagrams and dielectric properties of $\mathrm{PbZr}_{1-x} \mathrm{Ti}_{x} \mathrm{O}_{3}$ polydomain thin films, Phys. Rev. B 91 (14) (2015) 144106.

[42] R. J. Xu, J. Karthik, A. R. Damodaran, L. W. Martin, Stationary domain wall contribution to enhanced ferroelectric susceptibility, Nat. Commun. 5 (2013) 3120.

[43] R. J. Xu, S. Liu, I. Grinberg, J. Karthik, A. R. Damodaran, A. M. Rappe, L. W. Martin, Ferroelectric polarization reversal via successive ferroelastic transitions, Nat. Mater. 14 (2015) 79-86.

[44] C. S. Ganpule, A. L. Roytburd, V. Nagarajan, B. K. Hill, S. B. Ogale, E. D. Williams, R. Ramesh, J. F. Scott, Polarization relaxation kinetics and $180^{\circ}$ domain wall dynamics in ferroelectric thin films, Phys. Rev. B 65 (1) (2001) 014101.

[45] S. Bühlmann, E. Colla, P. Muralt, Polarization reversal due to charge injection in ferroelectric films, Phys. Rev. B 72 (21) (2005) 214120. 
[46] P. Gao, J. Britson, J. R. Jokisaari, C. T. Nelson, S. H. Baek, Y. R. Wang, C. B. Eom, L. Q. Chen, X. Q. Pan, Atomic-scale mechanisms of ferroelastic domain-wall-mediated ferroelectric switching, Nat. Commun. 4 (2013) 2791.

[47] P. Gao, J. Britson, C. T. Nelson, J. R. Jokisaari, C. Duan, M. Trassin, S. H. Baek, H. Guo, L. Li, Y. R. Wang, Y. H. Chu, A. M. Minor, C. B. Eom, R. Ramesh, L. Q. Chen, X. Q. Pan, Ferroelastic domain switching dynamics under electrical and mechanical excitations, Nat. Commun. 5 (2014) 3801.

[48] S. V. Kalinin, S. Jesse, B. J Rodriguez, J. Shin, A. P. Baddorf, H. N. Lee, A. Borisevich, S. J. Pennycook, Spatial resolution, information limit, and contrast transfer in piezoresponse force microscopy, Nanotechnology 17 (14) (2006) 3400.

[49] Z. J. Hong, J. Britson, J. M. Hu, L. Q. Chen, Local $90^{\circ}$ switching in $\mathrm{Pb}\left(\mathrm{Zr}_{0.2} \mathrm{Ti}_{0.8}\right) \mathrm{O}_{3}$ thin film: Phase-field modeling, Acta Mater. 73 (2014) 75-82.

[50] Y. L. Li, S. Y. Hu, L. Q. Chen, Ferroelectric Domain Morphologies of (001) $\mathrm{PbZr}_{1-x} \mathrm{Ti}_{x} \mathrm{O}_{3}$ Epitaxial Thin Films, J. Appl. Phys. 97 (3) (2005) 034112.

[51] Y. L. Li, L. Q. Chen, Temperature-Strain Phase Diagram for $\mathrm{BaTiO}_{3}$ Thin Films, Appl. Phys. Lett. 88 (7) (2006) 072905.

[52] J. X. Zhang, Y. L. Li, S. Choudhury, L. Q. Chen, Y. H. Chu, F. Zavaliche, M. P. Cruz, R. Ramesh, Q. X. Jia, Computer simulation of ferroelectric domain structures in epitaxial $\mathrm{BiFeO}_{3}$ thin films, J. Appl. Phys. 103 (9) (2008) 094111.

[53] J. J. Wang, J. M. Hu, T. N. Yang, M. Feng, J. X. Zhang, L. Q. Chen, C. W. Nan, Effect of strain on voltage-controlled magnetism in $\mathrm{BiFeO}_{3}$-based heterostructures, Scientific Reports 4 (2014) 4553.

[54] J. J. Wang, J. M. Hu, R. C. Peng, Y. Gao, Y. Shen, L. Q. Chen, C. W. Nan, Magnetization reversal by out-of-plane voltage in $\mathrm{BiFeO} 3$-based multiferroic heterostructures, Scientific Reports 5 (2015) 10459.

[55] J. C. M. Garnett, Colours in Metal Glasses and in Metallic Films, Philos. Trans. R. Soc., A 203 (359-371) (1904) 385.

[56] R. Landauer, Electrical conductivity in inhomogeneous media, AIP Conf. Proc. 40 (1978), 2.

[57] H. W. Jang, D. Ortiz, S. H. Baek, C. M. Folkman, R. R. Das, P. Shafer, Y. Chen, C. T. Nelson, X. Pan, R. Ramesh, and C. B. Eom, Domain Engineering for Enhanced Ferroelectric Properties of Epitaxial (001) BiFeO Thin Films, Adv. Mater. 21 (7) (2009) 817.

[58] Y. H. Chu, M. P. Cruz, C. H. Yang, L. W. Martin, P. L. Yang, J. X. Zhang, K. Lee, P. Yu, L. Q. Chen, and R. Ramesh, Domain Control in Multiferroic BiFeO3 through Substrate Vicinality, Adv. Mater. 19 (18) (2007) 2662.

[59] B. Winchester, P. Wu, and L. Q. Chen, Phase-field simulation of domain structures in epitaxial BiFeO3 films on vicinal substrates, Appl. Phys. Lett. 99 (5) (2011) 052903.

[60] M. Foeth, A. Sfera, P. Stadelmann, P.-A. Buffat, A comparison of HREM and weak beam transmission electron microscopy for the quantitative measurement of the thickness of ferroelectric domain walls, J. Electron Microsc. 48 (6) (1999) 717-723.

[61] X. Zhang, T. Hashimoto, D. C. Joy, Electron holographic study of ferroelectric domain walls, Appl. Phys. Lett. 60 (6) (1992) 784.

[62] K. S. Lee, J. H. Choi, J. Y. Lee, and S. Baik, Domain formation in epitaxial $\mathrm{Pb}(\mathrm{Zr}, \mathrm{Ti}) \mathrm{O}_{3}$ thin films, J. Appl. Phys. 90 (8) (2001) 4095-4102. 
[63] W. Pompe, X. Gong, Z. Suo, and J. S. Speck, Elastic energy release due to domain formation in the strained epitaxy of ferroelectric and ferroelastic films, J. Appl. Phys. 74 (10) (1993) 6012-6019.

[64] R. People, J. C. Bean, Calculation of critical layer thickness versus lattice mismatch for Ge ${ }_{x} \mathrm{Si}_{1-x} / \mathrm{Si}$ strained-layer heterostructures, J. Appl. Phys. 47 (3) (1985) 322-324.

[65] R. Cheaito, J. C. Duda, T. E. Beechem, K. Hattar, J. F. Ihlefeld, D. L. Medlin, M. A. Rodriguez, M. J. Campion, E. S. Piekos, P. E. Hopkins, Experimental Investigation of Size Effects on the Thermal Conductivity of Silicon-Germanium Alloy Thin Films, Phys. Rev. Lett. 109 (12) (2012) (195901).

[66] A. M. Marconnet, M. Asheghi, and K. E. Goodson, From the casimir limit to phononic crystals: Twenty years of phonon transport studies using silicon-on-insulator technology, Journal of Heat Transfer, 135 (2013) 061601.

[67] A. Gruverman, O Auciello, R Ramesh, H Tokumoto, Scanning force microscopy of domain structure in ferroelectric thin films: imaging and control, Nanotechnology 8, (3A) (1997) 38.

[68] S. V. Kalinin, A. N. Morozovska, L. Q. Chen, and B. J. Rodriguez, Local polarization dynamics in ferroelectric materials, Rep. Prog. Phys. 73 (5) (2010) 056502. 
(a)
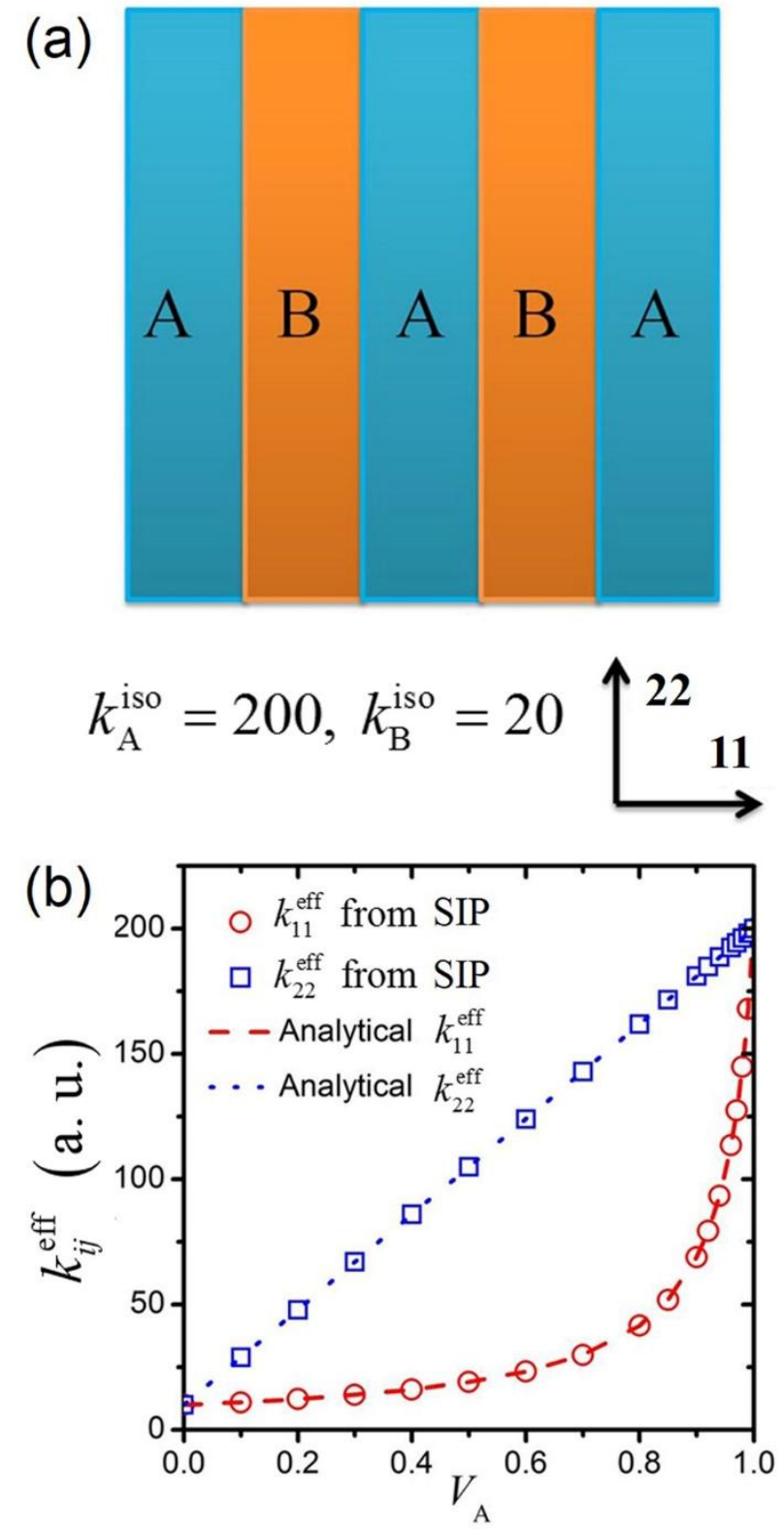

Figure 1. (a) Idealized two-phase microstructures composed of parallel slabs of A and B with isotropic but different thermal conductivity: $k_{A}^{\text {iso }}=200$ and $k_{B}^{\text {iso }}=20$ in same units. (b) Computed and theoretical effective thermal conductivity components as a function of volume fraction of phase A. 

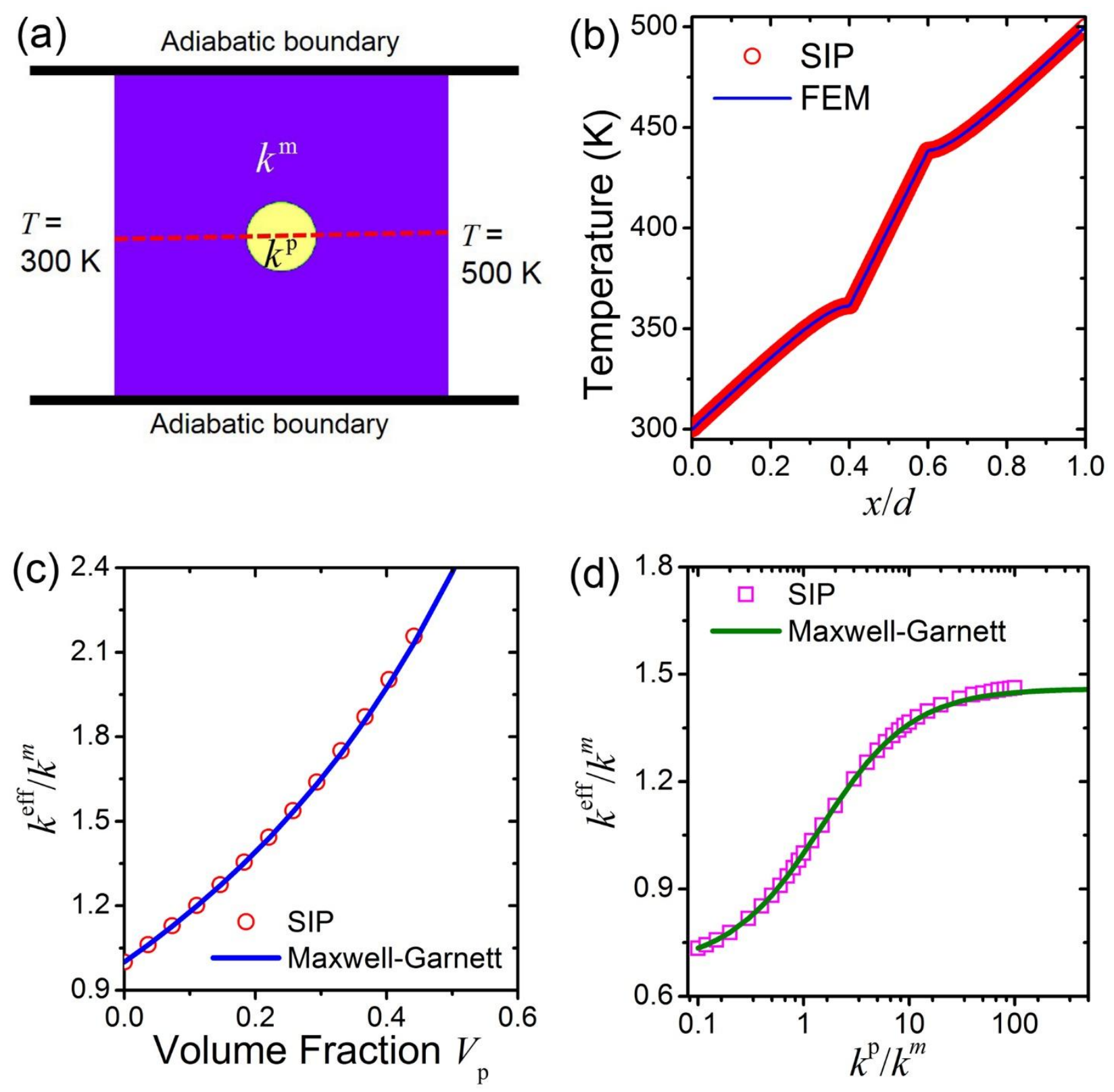

Figure 2. (a) Schematic illustration of a two-phase microstructure composed of a square matrix and a circular inclusion with isotropic thermal conductivities and specific boundary conditions. (b) The temperature distributions at the middle cross section of the composite (red dashed line in Fig. 2a) solved from the stationary heat conduction equation using the SIP method and finite element method (FEM), respectively. The effective thermal conductivity of this two-phase microstructure as function of (c) the volume fraction of the inclusion and (d) the ratio of $k^{\mathrm{p}}$ to $k^{\mathrm{m}}$, compared between the results obtained from the SIP method and the Maxwell-Garnett effective medium model. 

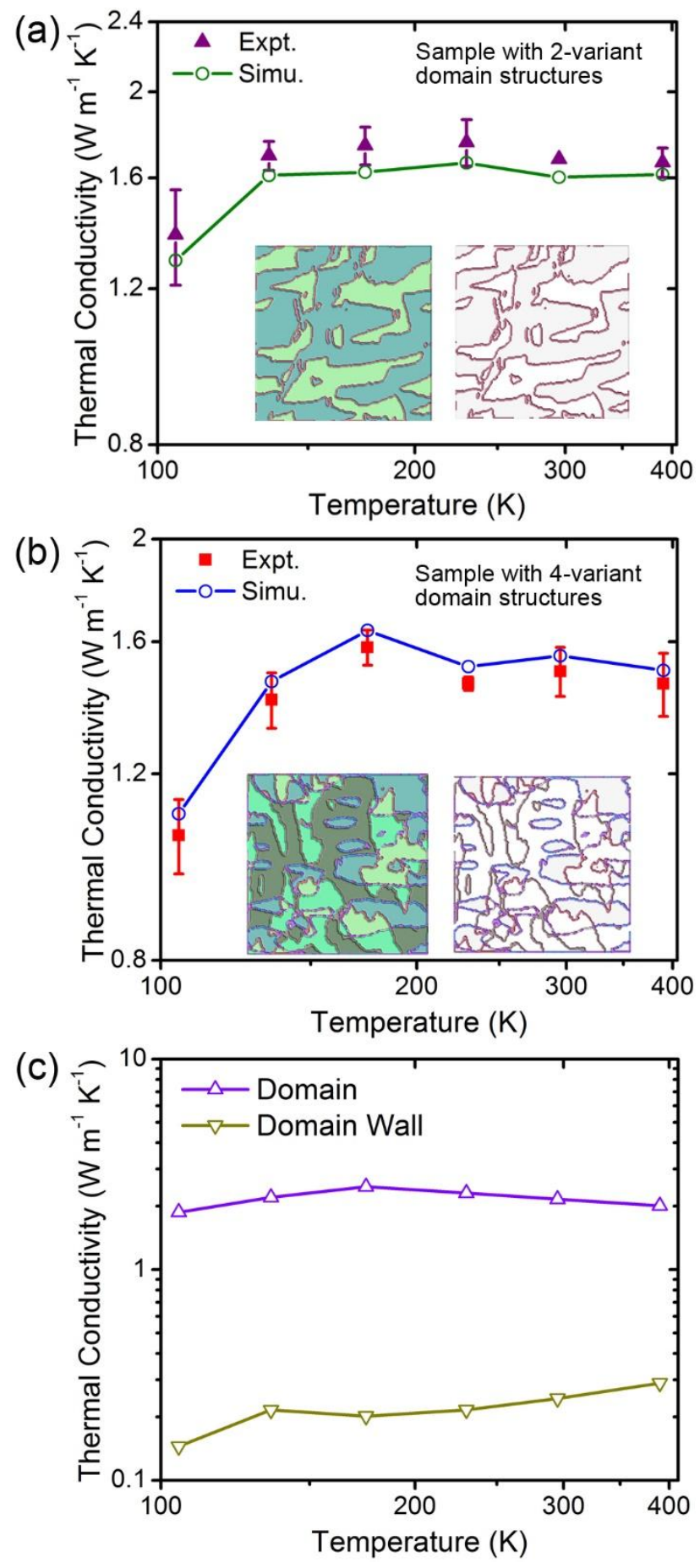

Figure 3. Experimental and simulated effective thermal conductivity of (001)-oriented $\mathrm{BiFeO}_{3}$ thin films with (a) 2-variant domain structures and (b) 4-variant domain structures. (c) Thermal conductivity of the domain and domain wall used as inputs for the simulation. The experimental data is from Ref. [20]. The polarization orientation in each domain indicated by different color is elaborated by the colorwheel of Fig.4c. 

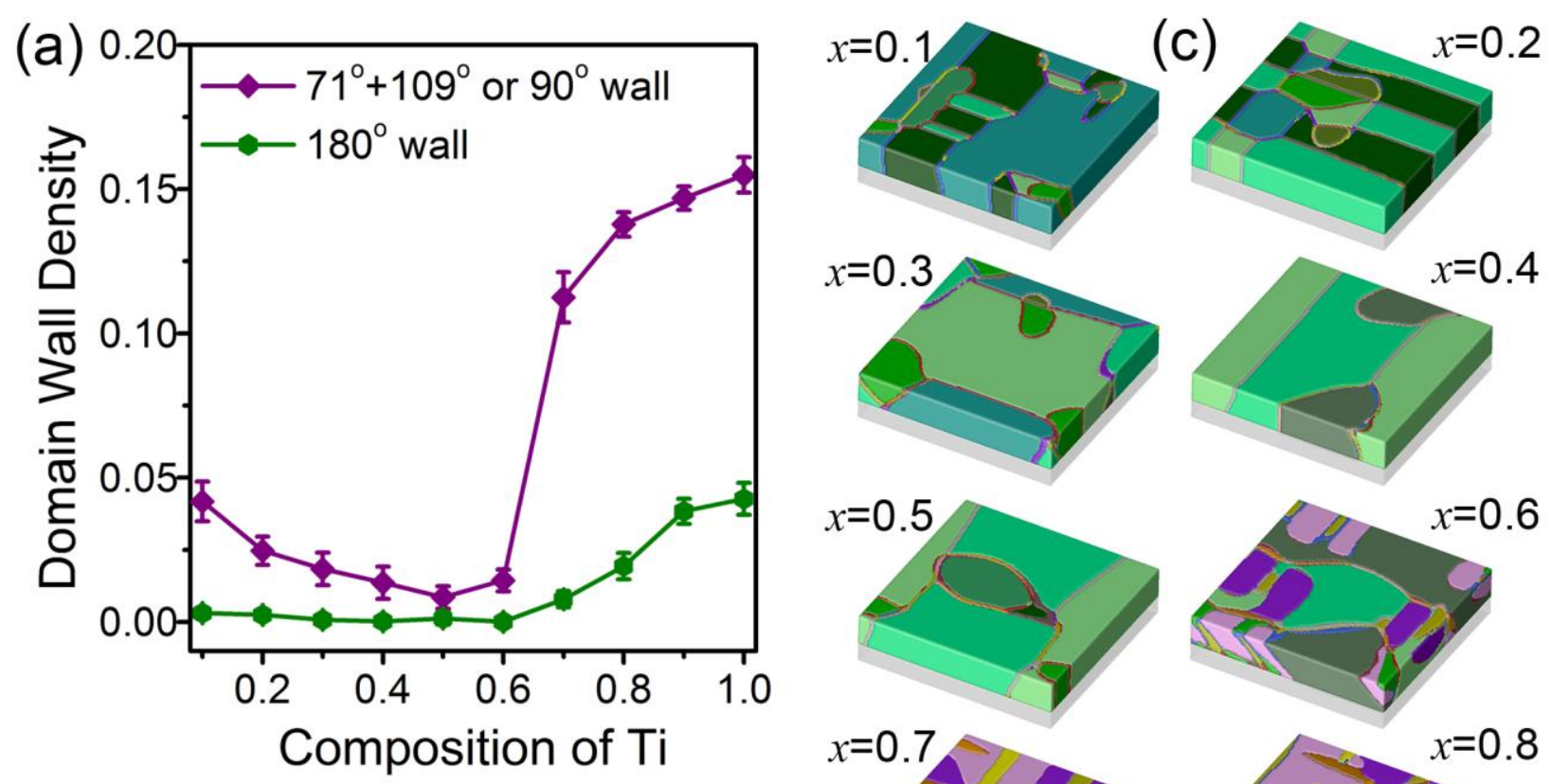

(b)
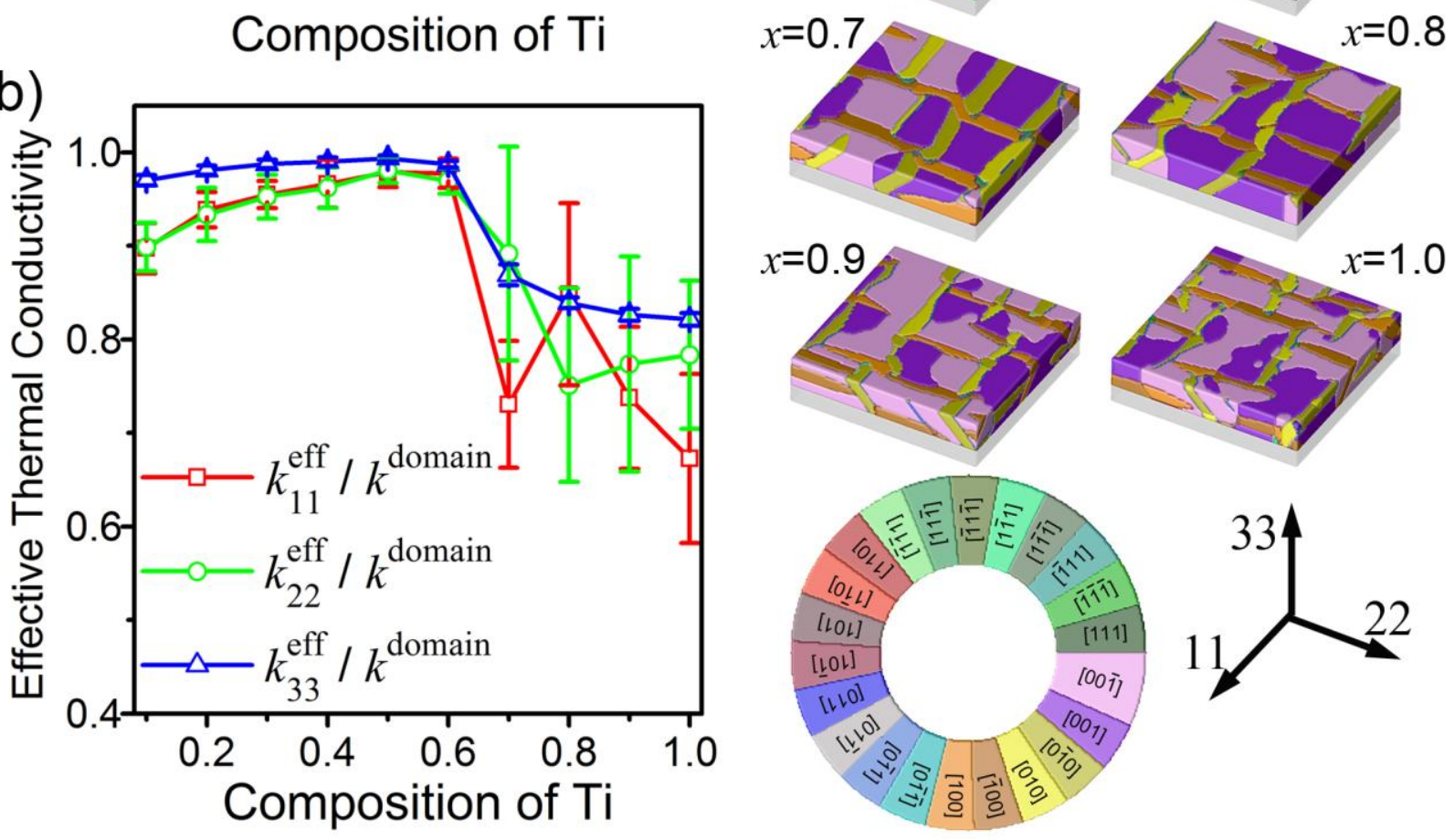

Figure 4. Composition-engineering (a) domain wall density, (b) effective thermal conductivity, and (c) domain structures of $\mathrm{PbZr}_{1-x} \mathrm{Ti}_{x} \mathrm{O}_{3}$ films clamped with zero misfit strain. The colorwheel in (c) shows the corresponding polarization orientation in domains denoted by different colors. The coordinate system of the domain structures is illustrated at the bottom right of (c). The symbols and bars in (a) and (b) represent the corresponding average values and standard deviations, respectively. 

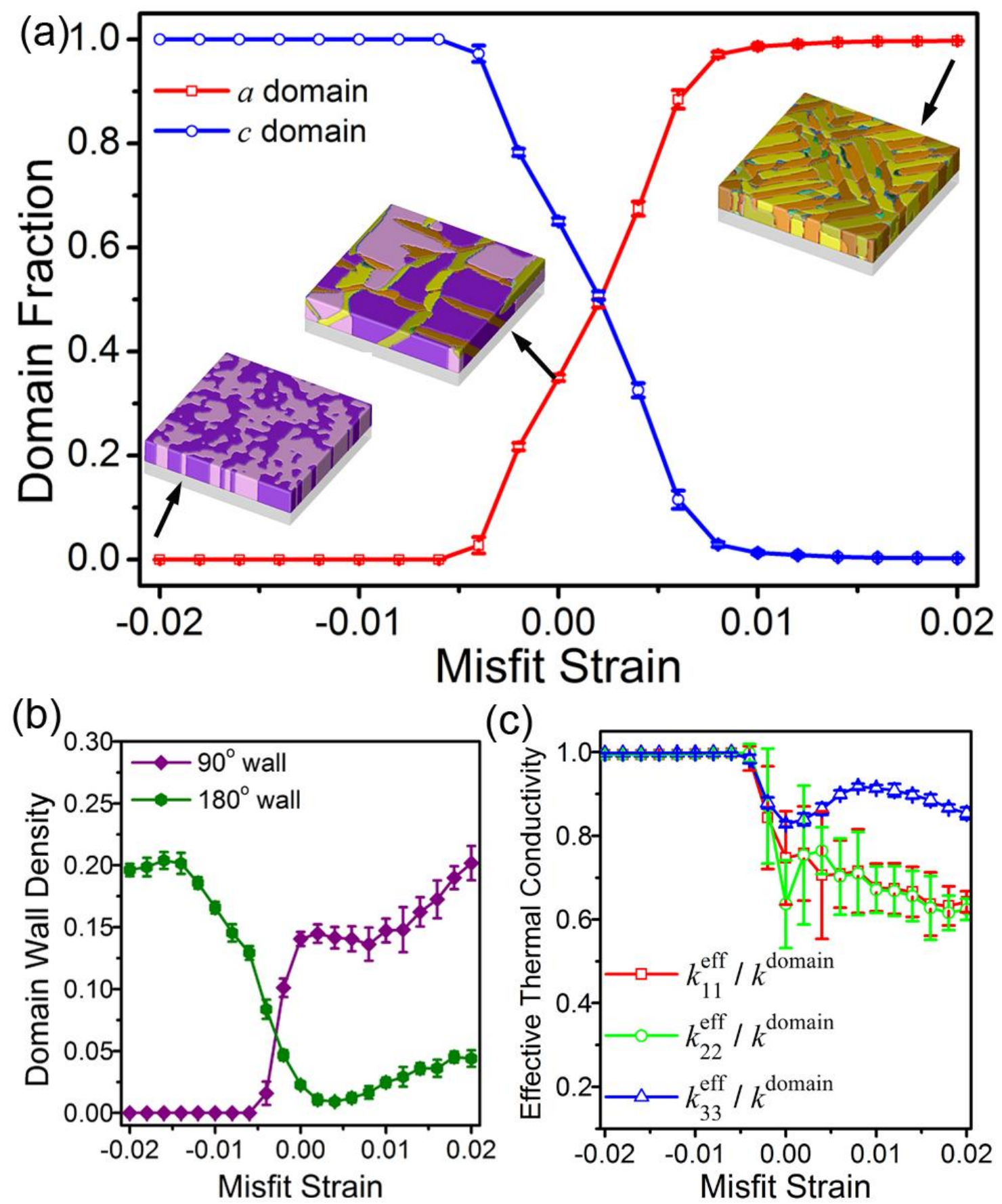

(c)

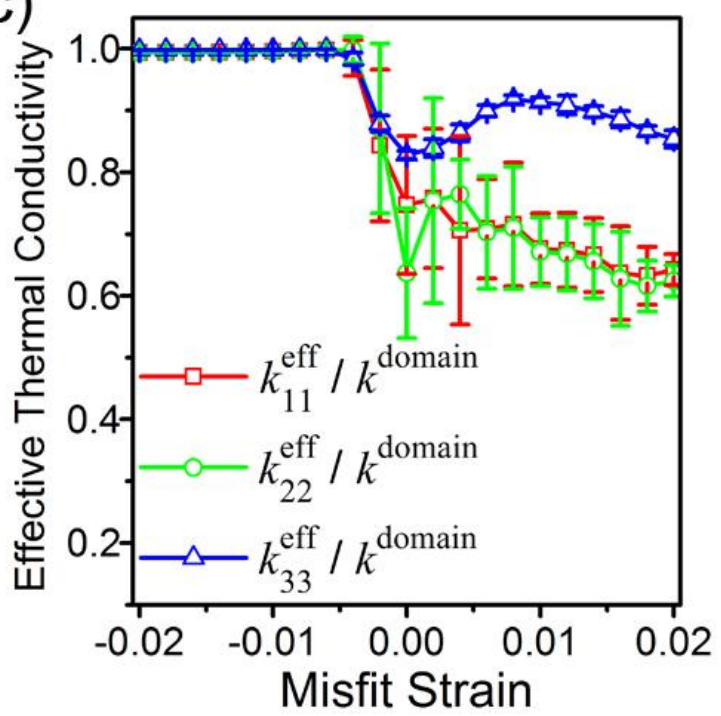

Figure 5. Misfit strain-engineering (a) domain structures, (b) domain wall density, and (c) effective thermal conductivity for $\mathrm{PbZr}_{0.2} \mathrm{Ti}_{0.8} \mathrm{O}_{3}$ films. The insets of (a) show the domain structures of $c$ domains, $a c$ domains and $a a$ domains. The symbols and bars in (a), (b), and (c) represent the corresponding average values and standard deviations, respectively. 
(a)

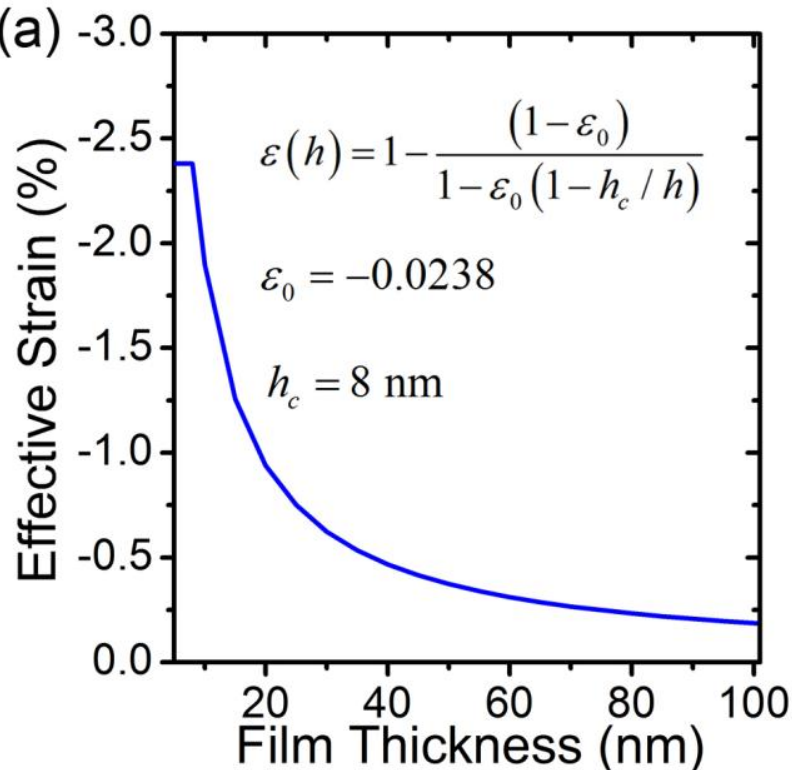

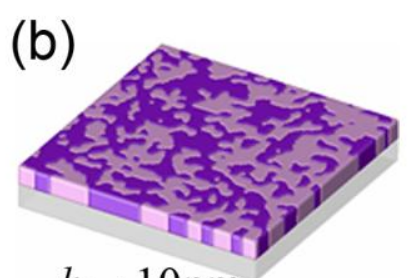

$h=10 \mathrm{~nm}$
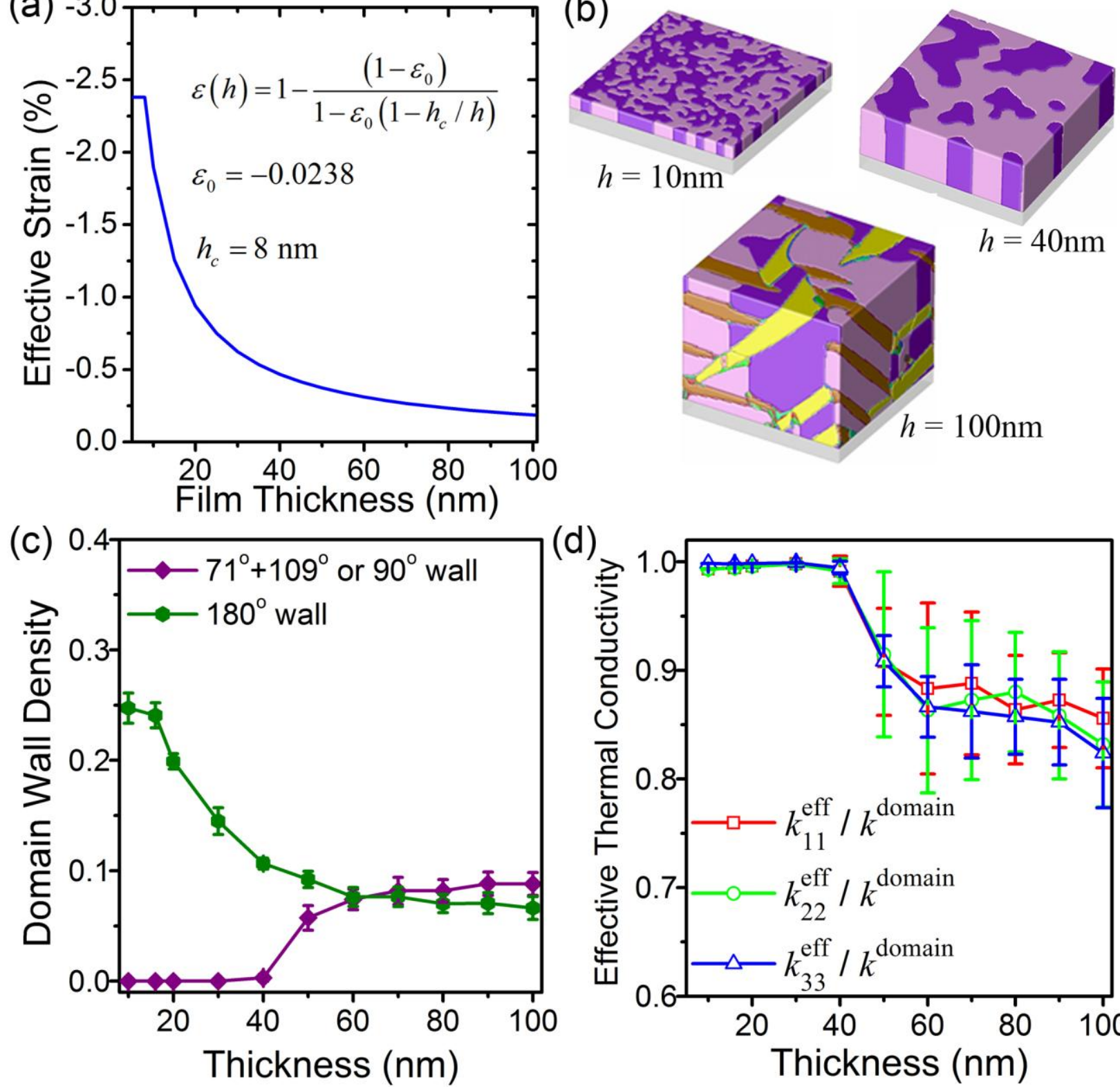

(d)

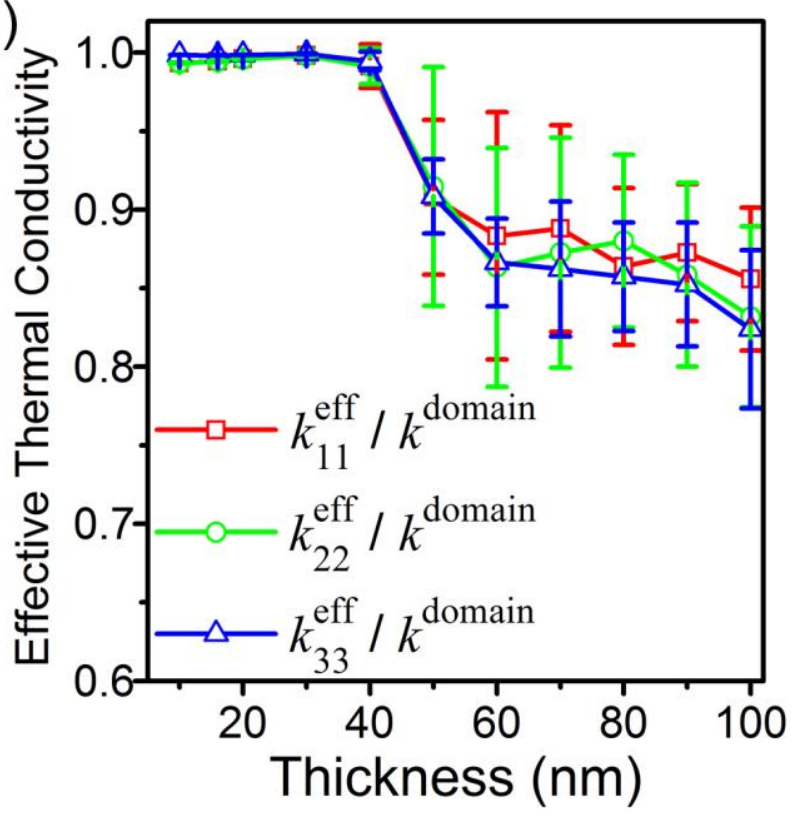

Figure 6. Film thickness-engineering (a) effective misfit strain, (b) domain structures, (c) domain wall density, and (d) effective thermal conductivity for (001)-oriented $\mathrm{PbZr}_{0.2} \mathrm{Ti}_{0.8} \mathrm{O}_{3}$ films grown on (001) $\mathrm{SrTiO}_{3}$ substrate. The effective strain as function of film thickness is calculated from the People-Bean (PB) model which predicts a critical strain of 8nm for the formation of dislocation. The symbols and bars in (c) and (d) represent the corresponding average values and standard deviations, respectively. 

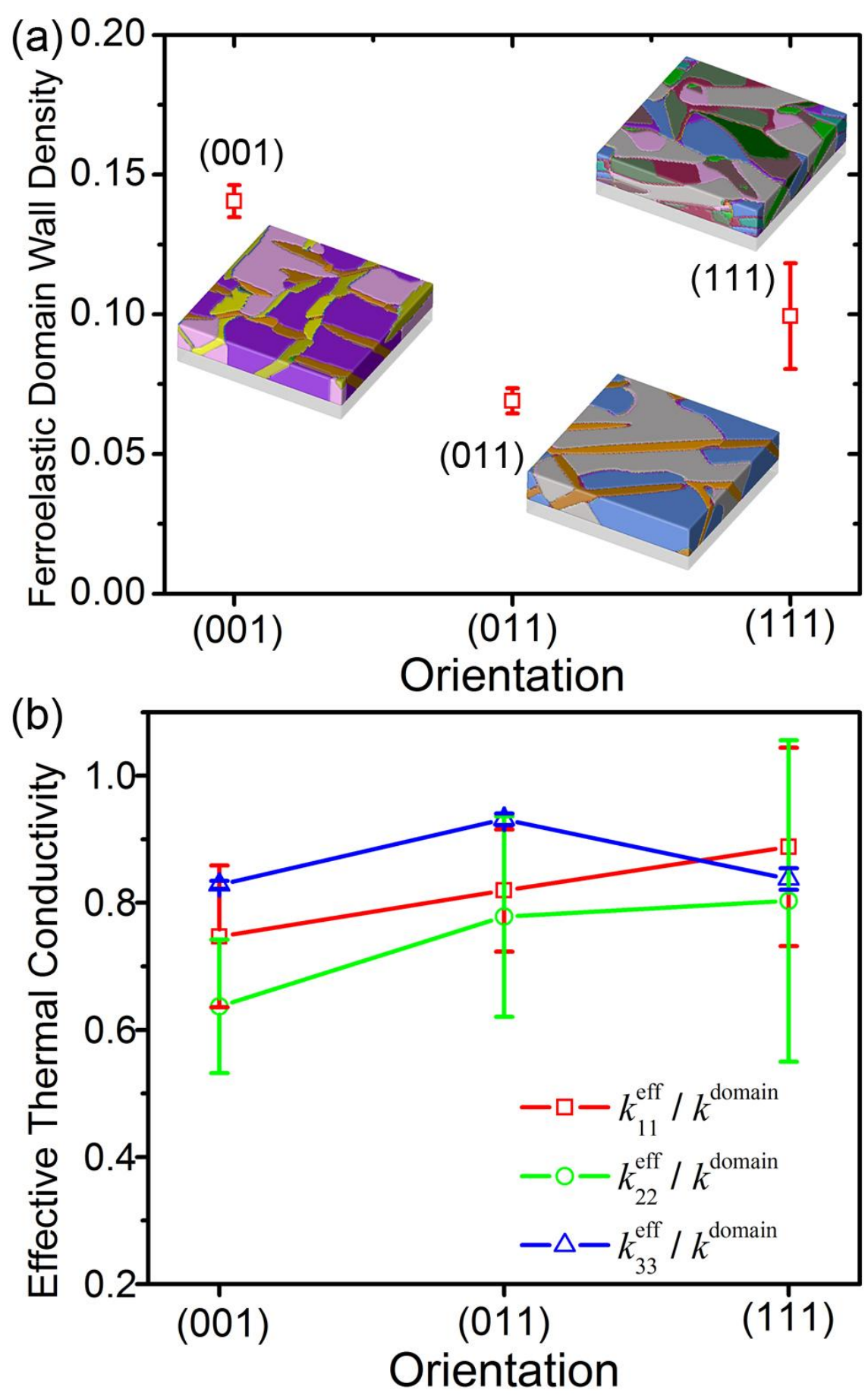

Figure 7. Orientation-engineering (a) domain wall density and domain structures, and (b) effective thermal conductivity for $\mathrm{PbZr}_{0.2} \mathrm{Ti}_{0.8} \mathrm{O}_{3}$ films grown on different oriented $\mathrm{SrTiO}_{3}$ substrate. Mismatch strains for these three films are assumed to be the same and with zero value. The polarization direction in domains with different colors are indicated by the colorwheel in Fig. 4c. The symbols and bars in (a) and (b) represent the corresponding average values and standard deviations, respectively. 

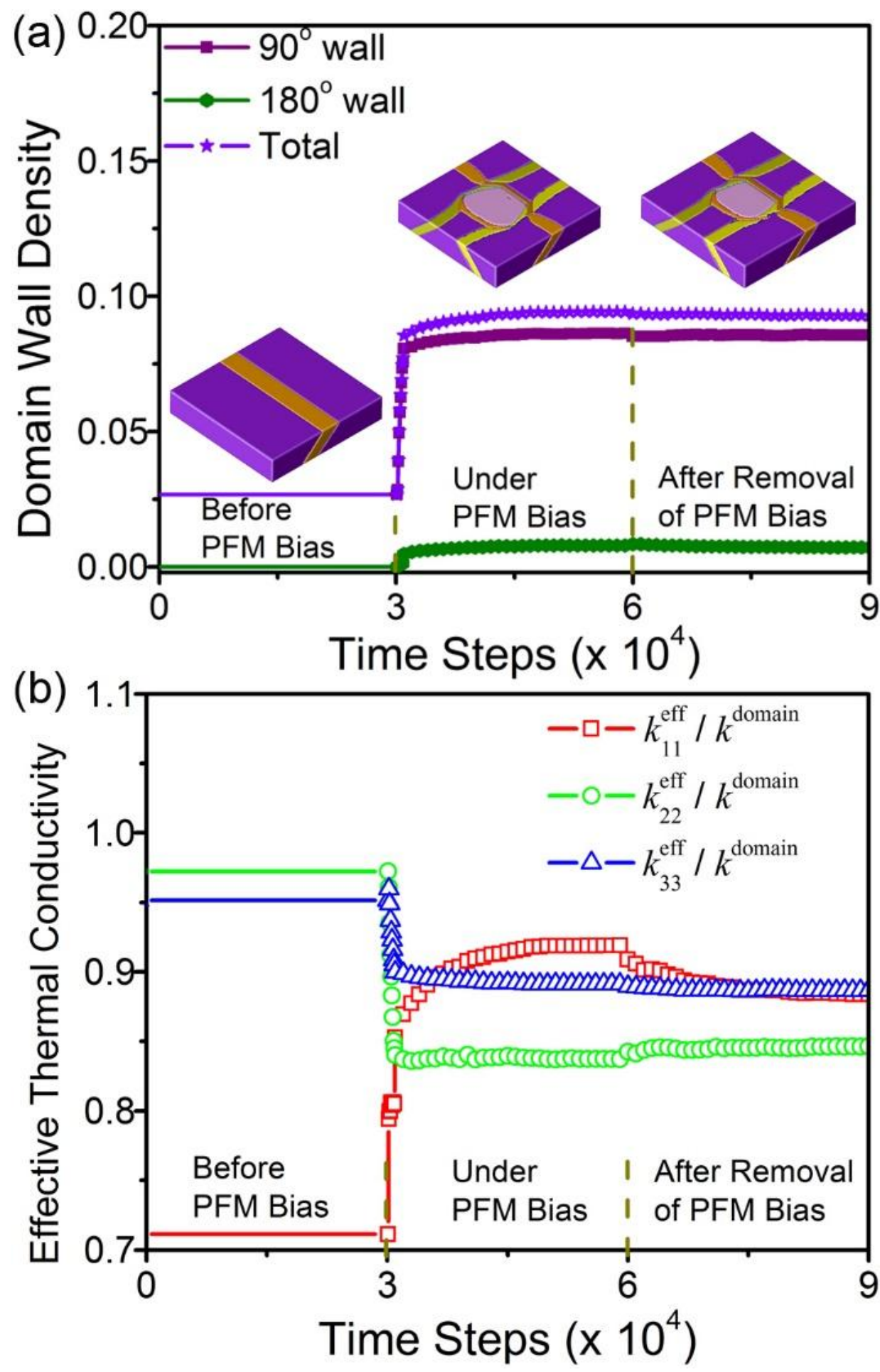

Figure 8. Piezoresponse Force Microscopy-tuning (a) domain wall density and domain structures, and (b) effective thermal conductivity for a (001)-oriented thin $\mathrm{PbZr}_{0.2} \mathrm{Ti}_{0.8} \mathrm{O}_{3}$ with mismatch strain of zero. The polarization direction in domains with different colors are indicated by the colorwheel in Fig. 4c. 

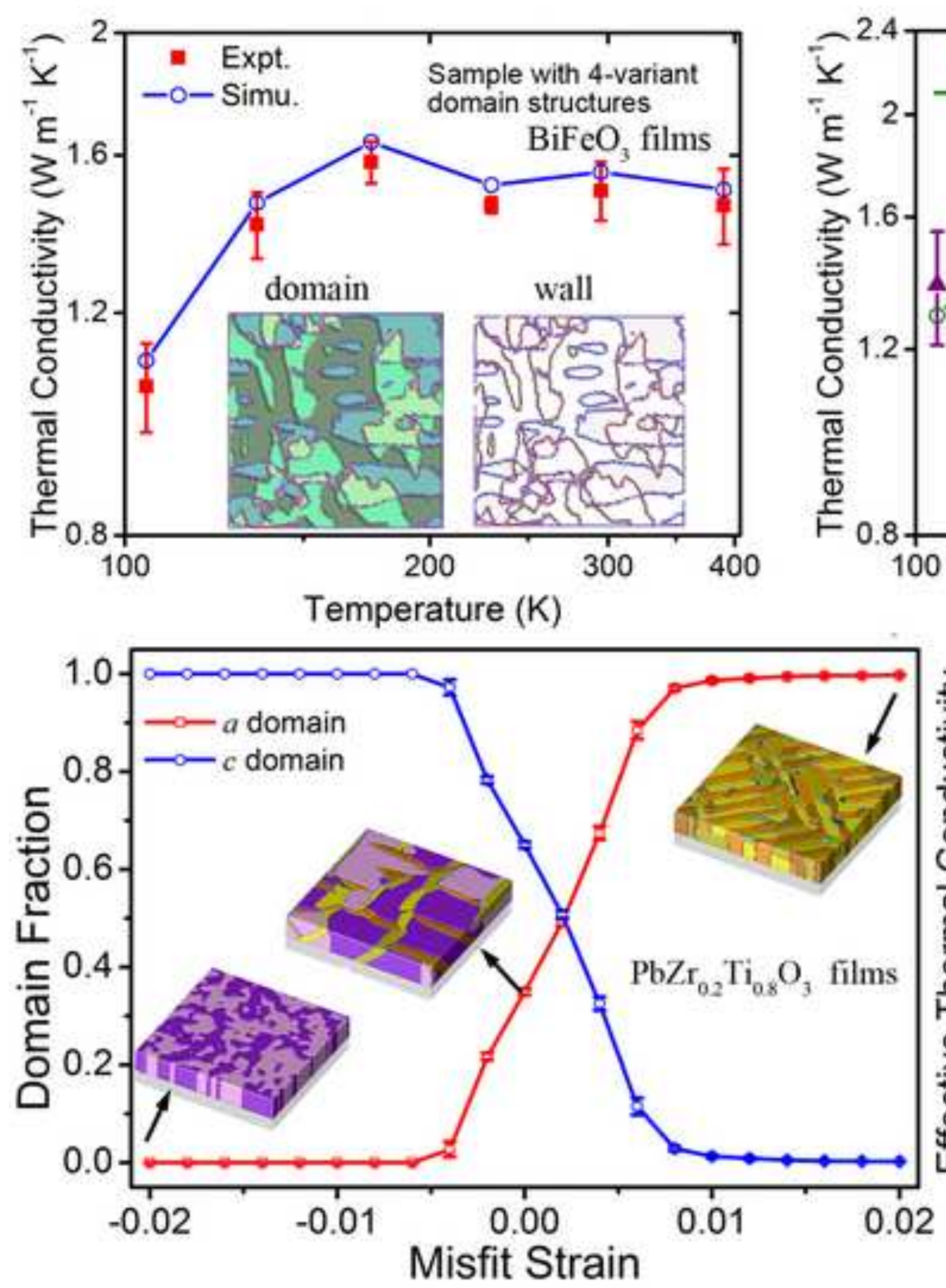
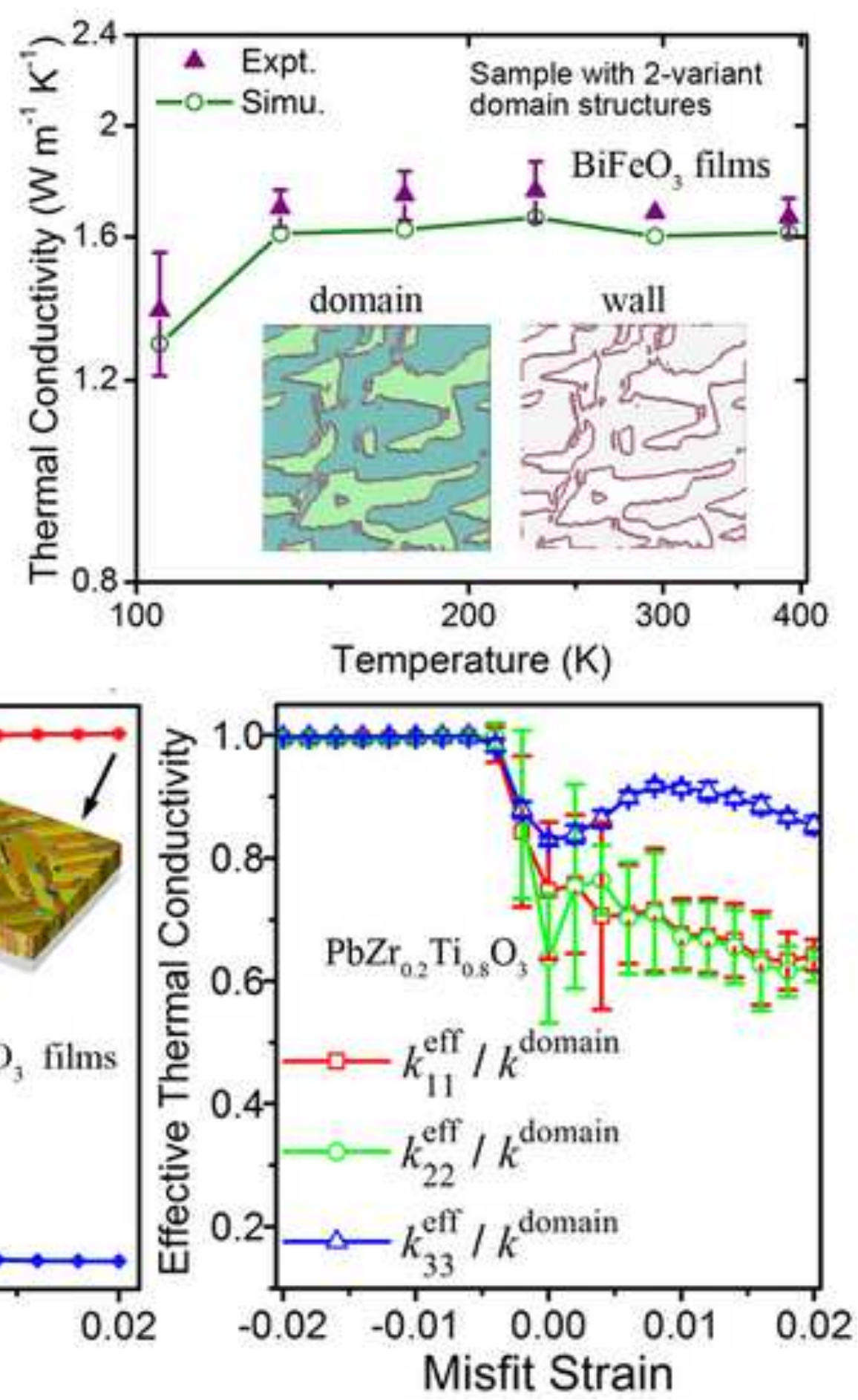